\title{
Linear stability analysis of axisymmetric perturbations in imperfectly conducting liquid jets
}

\author{
J. M. López-Herrera, ${ }^{\text {a) }}$ P. Riesco-Chueca, ${ }^{\text {b) }}$ and A. M. Gañán-Calvo ${ }^{c}$ \\ Escuela Superior de Ingenieros, Universidad de Sevilla, Camino de los Descubrimientos s/n, \\ Sevilla 41092, Spain
}

(Received 7 June 2004; accepted 7 January 2005; published online 1 March 2005)

\begin{abstract}
A discussion is presented on the role of limited conductivity and permittivity on the behavior of electrified jets. Under certain conditions, significant departures with respect to the perfect-conductor limit are to be expected. In addition, an exploration is undertaken concerning the validity of one-dimensional average models in the description of charged jets. To that end, a temporal linear modal stability analysis is carried out of poor-conductor viscous liquid jets flowing relatively to a steady radial electric field. Only axisymmetric perturbations, leading to highest quality aerosols, are considered. A grounded coaxial electrode is located at variable distance. Most available studies in the literature are restricted to the perfect-conductor limit, while the present contribution is an extension to moderate and low electrical conductivity and permittivity jets, in an effort to describe a situation increasingly prevalent in the sector of small-scale free-surface flows. The influence of the electrode distance $b$, a parameter $\alpha$ defined as the ratio of the electric relaxation time scale to the capillary time scale, and the relative permittivity $\beta$ on the growth rate has been explored yielding results on the stability spectrum. In addition, arbitrary viscosity and electrification parameters are contemplated. In a wide variety of situations, the perfect-conductor limit provides a good approximation; however, the influence of $\alpha$ and $\beta$ on the growth rate and most unstable wavelength cannot be neglected in the general case. An interfacial boundary layer in the axial velocity profile occurs in the low-viscosity limit, but this boundary layer tends to disappear when $\alpha$ or $\beta$ are large enough. The use of a one-dimensional (1D) averaged model as an alternative to the 3D approach provides a helpful shortcut and a complementary insight on the nature of the jet's perturbative behavior. Lowest-order 1D approximations (average model), of widespread application in the literature of electrified jets, are shown to be inaccurate in low-viscosity imperfect-conductor jets. (C) 2005 American Institute of Physics. [DOI: 10.1063/1.1863285]
\end{abstract}

\section{INTRODUCTION}

Electrification of free-surface flows is guided by three principal aims: first, increasing the surface-to-volume ratio by means of a reduction of the liquid's apparent surface tension; second, controlling the stability range of the flow; third, directing the flow towards a target. Multiple applications derive from these achievements: atomization, particle sorting, ink jet printing, fuel injection, and fiber spinning. For instance, jet stimulation by electric fields is increasingly used in a variety of ink jet printing technologies as a method for charging and steering the ink drops resulting from jet disintegration. Alternatively, continuous deviated ink jet technology is based on direct deflection of electrified jets. Electric charges modulate the jet's response to electric fields, random noise, or specific excitation wavelengths. In many applications, a grounded electrode located at the vicinity of the jet surface establishes a radial field; the quasielectrostatic pressure jump competes with convective, capillary, and viscous effects to determine the selected wavelength. Another research field involving electrified jets is electrospinning, a process in which solid fibers are produced from a polymeric

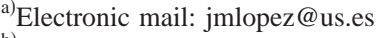

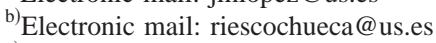

${ }^{\mathrm{c})}$ Electronic mail: amgc@us.es
}

fluid stream delivered through a millimeter-scale nozzle. Additional achievements are presently reported in pharmaceutical and material sciences. These applications are leading to a growing interest of researchers in electrified jets. In the search for diverse applications, poor conductivity liquids are becoming increasingly attractive, because food and pharmacy technologies, among others, depend on their efficient handling.

An important motivation source for the study of electrohydrodynamics is the search for high quality sprays from liquid microjets, eagerly demanded in technological applications requiring small and homogeneous droplet size. The axisymmetric capillary breakup (Rayleigh breakup) of small and steady liquid ligaments into droplets gives rise to controllable monodisperse aerosols, unattainable through other high-yield processes.

The foundations for the study of conducting jets in a radial electric field were set by Melcher, ${ }^{1}$ assuming inviscid flow and constant electric potential. Saville ${ }^{2}$ generalized the analysis for arbitrary viscosity liquids. Huebner and $\mathrm{Chu}^{3}$ studied the stability of a charged jet in the inviscid limit assuming arbitrary electrode distance $b$. Setiawan and Heister $^{4}$ extended this description to the nonlinear growth phase. The influence of the surrounding atmosphere on the stability of the jet was considered by Baudry et al. ${ }^{5}$ An el- 
egant overview of the linear stability problem was presented by García, ${ }^{6}$ who explored the perturbative spectrum for a variety of parameters. This work has been recently extended to ac electric fields by González et al. $;^{7}$ the authors provide an attractive formulation of the dc problems, whose notation we follow here.

The electrical behavior of a moving liquid follows from the ratio of the characteristic hydrodynamic time versus the electrical relaxation time $t_{e} \sim \varepsilon_{i} / K$, where $\varepsilon_{i}$ and $K$ are the electrical permittivity and conductivity of the liquid, respectively. In the absence of other forcing agents, the time scale on which perturbations grow and cause the jet's breakup is given by a balance of surface tension and inertia, summed up by the capillary time $t_{c} \sim\left(\rho A^{3} / \gamma\right)^{1 / 2}$, where $\rho, \gamma$, and $A$ are the liquid density, surface tension, and the jet radius, respectively. If the ratio $t_{e} / t_{c}$ is strictly zero the liquid can be considered as a perfect conductor even in the last stages of pinch-off. In the perfect-conductor limit the calculation of electrical stresses on the jet surface is simplified since all charges are interfacial, the external electric field is normal to it, and the electric field in the jet bulk is zero. In this limit, provided the breakup is axisymmetric, electrification stabilizes long-wavelength perturbations while destabilizing short ones. $^{1,8}$

In the present work we aim to study the stability of electrified liquid jets when the ratio $t_{e} / t_{c}$ is not strictly zero. Our model is directly borrowed from the general formulation Saville provided in 1997 by expanding previous results from Taylor and Melcher. ${ }^{9-11}$ The analysis is applicable to situations where the relaxation time for free charges is short compared with the time scale for fluid motion. In the survey published in 1997 by Saville, ${ }^{9}$ the stability of fluid cylinders or free jets and pinned cylinders or liquid bridges is studied. These examples are given as an illustration of the powerful set of equations for the leaky dielectric model, which builds the core of the above survey. The discussion is mostly qualitative, and the results disclosed are restricted to some particular cases where a solution can be obtained with relative ease. Additional contributions in this field by Saville and co-workers focused on the effect of axial electric forcing. ${ }^{2,12-15}$

The starting point is provided by the linearized NavierStokes equations. A careful description is then made of the parametrical behavior of the resulting dispersion equation. Our study attempts to complete a systematic consideration of all the input variables (wavelength, viscosity, surface tension, electrical conductivity, permittivity and electrification levels, surrounding gas dynamics). Previous works do not include all these terms or do not weigh their relative influence. The ambient influence is explored by means of a sketchy model, which does however allow a preliminary glimpse into the stability of electrified coflowing liquid-gas streams. The simultaneous forcing of a jet by hydrodynamic focusing (flowfocusing technique ${ }^{16}$ ) and by electrification is a different field of research, with a variety of presumed applications, e.g., ultrafine atomization; the present contribution may provide a stepping stone for the joint analysis of electrification and flow focusing.

In addition, under sufficiently general conditions, a one- dimensional model following López-Herrera et al. ${ }^{17}$ yields excellent results [for a comparison on the suitability of either a three-dimensional (3D) axisymmetric or a 1D model see for example Yildirim and Basaran $\left.{ }^{18}\right]$, which allows the detailed and quite inexpensive exploration of extense parametrical ranges of interest. It is our aim to determine under which conditions the lowest-order 1D approximation (sliceaverage model), widely used in the literature, is to be trusted. It can be anticipated that the assumption of a flat axial velocity profile is inaccurate when either of two situations occur: (a) the velocity profile is markedly convex; (b) an axial velocity boundary layer develops, due to the action of shear, at the interface. In both cases, the flat-profile approximation fails to provide a trustworthy description. It will be shown that jets with moderate conductivity and permittivity often display sharply convex or boundary-layer velocity profiles. Indeed, nonuniform radial profiles are favored by the combination of small viscosity, poor conductivity and permittivity, and a shear agent. Tangential stress may be generated by a variety of mechanisms, such as surfactant diffusion or, in our case, an electric field. Axially oriented electric fields are the most efficient shear agents and they lead to the conversion of electric potential into kinetic energy; but even radial fields as studied here can give rise to significant shear provided $\alpha$ and $\beta$ are small or moderate. It is important, therefore, to be cautious when modeling electrified jets: slice-average models may turn out to be inaccurate, so that a 3D model or higher-order expansions (parabolic) become unavoidable.

Moderate liquid conductivity and permittivity is readily observed in many technological fields, in particular whenever oils or other organic compounds are manipulated. Other practical applications, such as electrospinning or jet printing, may involve imperfect conductors. In the following section, once the main dimensionless groups are introduced, reference will be made to experimental and technological applications involving low conductivity/permittivity liquids.

\section{FORMULATION OF THE PROBLEM}

\section{A. Context and assumptions}

The results presented here arise from some previous work on the conditions leading to jet breakup, under a diversity of geometrical and electrification contexts. LópezHerrera and Gañán-Calvo ${ }^{19}$ presented a detailed experimental procedure concerning charged capillary jet breakup, in the assumption of perfect-conductor behavior and radial electric forcing. In the course of experimentation, an extended exploration was attempted of the poor-conductor range. As stated above, food, pharmacy, carburation, and other applications at hand are increasingly pushing researchers toward the study of poor-conductors' stability and breakup.

Preliminary experimental results provided a wide diversity of data leading to considerable trouble in the effort to calibrate and sort out raw data from a theoretical point of view. Therefore, a detailed theoretical description of poorconductor jet behavior appeared to be desirable, both to provide a solid basis for the experimental program, and to orientate the production of an efficient numerical code able to include breakup. As an intermediate goal, the stability analy- 
sis of a liquid jet is hereby described: it yields inference on (a) the size of drops after breakup and the stimulation required therefore; (b) the conditions required in the opposite case, i.e., when breakup is to be avoided and the integrity and longevity of the jet is to be preserved. Both objectives are technologically relevant.

We limit the scope of our study to the axisymmetric stability of capillary cylindrical jets of a liquid with density $\rho$, permittivity $\varepsilon_{i}$, viscosity $\mu$, conductivity $K$, and interfacial surface tension $\gamma$. Our interest will be focused on the axisymmetric capillary jet instability. This choice is dictated by two main reasons: first, the prevalence of axisymmetric instability in most applications where a high quality spray is required (e.g., when electrospraying small liquid flow rates). Second, axisymmetric modes are dominant in most dc estimulation regimes provided the Weber number is moderate. Accordingly, we restrict our study to moderate We numbers and ignore sinuous or asymmetric perturbation modes.

The surrounding atmosphere is assumed inviscid and its influence on the dynamics of the jet is modeled with the help of a simplified approach, which will be justified later by invoking some restrictions on the Weber numbers. $A$ and $U_{o}$ are the jet radius, assumed constant, and the average axial velocity. The equilibrium velocity profile is assumed flat; its value $U_{o}$ is usually scaled with the capillary wave velocity $v_{c}=(\gamma / \rho A)^{1 / 2}$ by means of the Weber number, We $=\left(\rho A U_{o}^{2}\right) / \gamma$.

In the gas-at-rest limit, where $U_{\infty}=0$, and assuming We $\gg 1$, Keller et $a l .{ }^{20}$ show that the perturbation is rapidly convected downstream with the jet velocity, thus avoiding the simultaneous propagation of disturbances in the upstream and downstream directions (absolute instability). See also Artana et $a .^{21}$ for a discussion on the role of absolute and convective instabilities in the evolution of electrified jets, as well as O'Donnell et al. ${ }^{22}$ and Chauhan et al. ${ }^{23}$ for an experimental investigation of the convective instability in uncharged jets. Kalaaji et al. ${ }^{24}$ presented an experimental investigation of the breakup length of a jet and showed that provided the Weber number is large enough, temporal and spatial linear perturbation theories coincide. We therefore restrict our study to moderate or large Weber numbers [approximately above 4, see Eq. (5) in Lin and Reitz ${ }^{25}$ ]: this restriction ensures that the jet velocity is sufficiently higher than the group velocity of perturbations so that a temporal evolution of the perturbation is observed from a Galilean reference frame moving with the jet. However, when the Weber number exceeds a critical value, either nonsymmetric perturbations grow faster than axisymmetric perturbations or viscous effects in the surrounding atmosphere can no longer be neglected.

The above references do not consider the coflowing regime, where both the jet and the surrounding gas flow (with different mean velocity) in the axial direction. This flow configuration is increasingly attractive as a source of potential technical applications. Unfortunately, the parametric limits of axisymmetric breakup in coflowing jets have not been explored in detail in terms of the liquid and gas Weber numbers. A mapping of the breakup regimes as sketched by Lin and Reitz ${ }^{25}$ in the gas-at-rest limit is not available in the coflowing case. It is known that interfacial shear promotes Taylor's instability, where droplets much smaller than the jet diameter are produced, ${ }^{26}$ but a systematic exploration of the breakup regimes prevailing under different gas and liquid Weber numbers is not available.

In the following, however, we narrow the scope of our attention to flow configurations located in the Rayleigh and first wind-induced regimes, where shear effects from the gas side can be ignored. Accordingly, aerodynamic effects (gasliquid interaction) are modeled with a simplified theory; the influence of the gas is accounted for in terms of its pressure fluctuation, but gas viscosity is neglected. The surrounding gas is assumed to flow coaxially with an uniform speed $U_{\infty}$ which, depending on the envisaged application, may range from 0 (ambient gas at rest) to an amount one or two orders of magnitude above the jet velocity $U_{o}$. With this choice, we leave the door open to the study of electrified flow focusing configurations (EF3), a field of considerable interest. The flow focusing technology is based on the acceleration of a liquid jet surrounded by a coflowing gas stream, whereby a favorable pressure gradient is created, allowing an enhanced control of the problem's hydrodynamics. ${ }^{16}$

Three main simplifications are introduced in our gasliquid interaction model.

(a) The gas is incompressible: results can be extrapolated to the case where the surrounding stream is also liquid, under the stringent condition that it be perfectly insulating.

(b) The gas is inviscid, and a uniform flow is assumed (flat axial velocity profile $U_{\infty}$ ).

(c) The surrounding stream is an insulator with vacuum permittivity: it removes all space charges and is perfectly unelectrified.

Therefore, the ensuing description will not undertake the analysis of the velocity boundary layer at the ambient side. A detailed investigation of the gas velocity profile is performed by Gordillo et al., ${ }^{27}$ but their results (the liquid jet dynamics is assumed inviscid; no electric effects are considered) cannot be extrapolated here.

Additional assumptions in our model ${ }^{9}$ are the following: magnetic fields generated by moving charges can be neglected (electrostatic assumption); gravitational acceleration is ignored.

Figure 1 shows the geometric outline of the problem. Initially, a potential difference $V_{o}$ is applied between the jet and a coaxial cylindrical electrode of radius $R_{\infty}=b A$ surrounding it. In this geometrical configuration, the axial electric field is negligible compared to the normal electrical field, as is the case in jets emitted by electrospray. ${ }^{28,29}$ The radial electric field creates, in the unperturbed state, a surface charge density located at the interface, the liquid bulk being electrically neutral.

In most of the applications, charged jets issue from a liquid source, where a voltage $V_{o}$ is applied, and end up at the breakup region. A transition from conduction to convection, as the relevant charge transport mechanism, takes place in the vicinity of the needle. Charges relax from the bulk to 


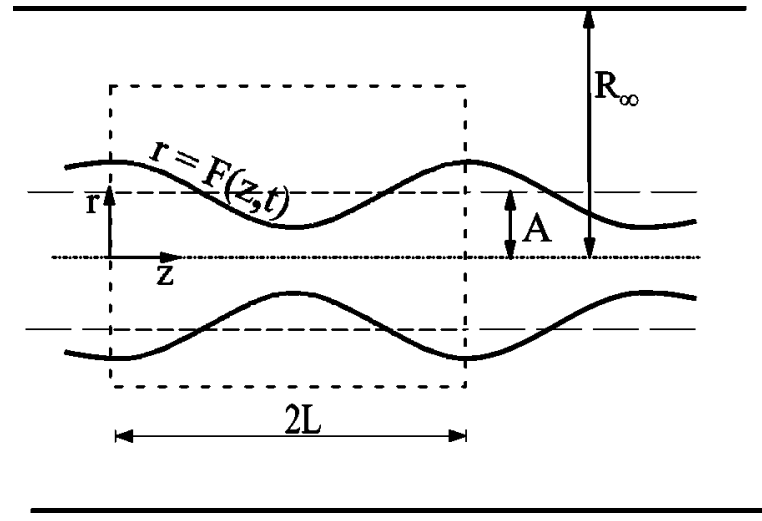

FIG. 1. Geometrical sketch of the problem.

the surface in a characteristic time $t_{e}$; this process taking place in a region of length $L_{r} \sim U_{o} t_{e}$ much shorter than the total jet length $L_{j}$. The jet length is generally of the order of $U_{o} t_{c}$, but in many practical situations, the longevity of the jet can be extended by avoiding all instability factors. Most of the voltage drop along the jet $\Delta V$ is located in the charge relaxation region, which can be modeled as a resistor of order $\Omega_{R} \sim L_{r} /\left(K \pi A^{2}\right)$; therefore, the characteristic relative voltage drop is

$$
\frac{\Delta V}{V_{o}} \sim \frac{\Omega_{R} I}{V_{o}} \sim \Omega_{R} \varepsilon_{o} U \sim \frac{\mathrm{We}}{\beta}\left(\frac{t_{e}}{t_{c}}\right)^{2} \ll 1,
$$

where $I$ is the current transported by the jet. Thus, the axial voltage drop in the relaxation stretch is negligible, and the potential at the jet surface can be estimated, in first approximation, as the potential applied at the needle $V_{o}$. Further insight on the structure of the potential drop along the axial coordinate of a jet is provided by Gamero-Castaño and Hruby $^{30}$ and Higuera. ${ }^{31}$

The equations of the problem are made dimensionless with the radius of the unperturbed jet $A$, the density $\rho$, surface tension $\gamma$, and the characteristic surface charge density $\varepsilon_{o} E_{o}, \varepsilon_{o}$ being the vacuum permittivity and $E_{o}$ $=V_{o} /[A \ln (b)]$ the characteristic radial electric field, while $b=R_{\infty} / A$. Our choice of $E_{o}$ rather than $V_{o}$ as a scaling factor has the following implication: at the interface, the normalized electrostatic pressure is of $O(1)$. This is unrealistic when $b \rightarrow \infty$, because an infinite potential difference $V_{o}$ is required in order to preserve the scaling; however, the results are consistent, and can be applied safely for any $b \gg 1$. The advantage in choosing $E_{o}$ rather than $V_{o}$ is that the normalized electrostatic pressure $\varepsilon_{o} E_{o}^{2}$ does not become vanishingly small in the $b \rightarrow \infty$ limit. Setiawan and Heister, ${ }^{4}$ who used the $V_{o}$ scaling, alluded to the shortcomings of this choice: "as the ground location is moved far from the jet, the effect of electrostatic contributions vanishes." Note, however, that $E_{o}$ must be bounded in our problem; otherwise, dielectric rupture would lead to corona discharge effects.

The above scaling leads to a set of nondimensional parameters which characterize each particular jet and perturbation.
(1) The half wavelength of the perturbation $\lambda=L / A$ or the dimensionless wavenumber $k=\pi / \lambda$.

(2) The radial position of the ground electrode $b=R_{\infty} / A$.

(3) The Weber number $\mathrm{We}=\rho A U_{o}^{2} / \gamma=\left(U_{o} / v_{c}\right)^{2}$, where $v_{c}$ $=(\gamma / \rho A)^{1 / 2}$ is the capillary velocity, measuring the relative importance of the jet's absolute inertia with respect to the capillary forces. Some authors prefer to use its inverse, which is referred to as a Euler number.

(4) An external flow Weber number $\mathrm{We}_{\infty}=\rho A U_{\infty}^{2} / \gamma$ $=\left(U_{\infty} / v_{c}\right)^{2}$, measuring the relative importance of the ambient fluid's absolute inertia with respect to the capillary forces. Note that the liquid's density (and not the gas') is used. In many applications, the ambient gas is at rest, so that $\mathrm{We}_{\infty}=0$. An additional parameter of interest in describing the liquid-gas interaction is the ratio between the gas and liquid densities $\bar{\rho}=\rho_{g} / \rho$.

(5) The Ohnesorge number $C=\mu /(\rho \gamma A)^{1 / 2}=\nu t_{c} / A^{2}$ weighing viscous forces against capillary forces. It is the inverse of a Reynolds number based on the capillary velocity; it can also be understood as the ratio between the capillary time $t_{c}$ and the viscous radial diffusion time $t_{v} \sim A^{2} / \nu$. The usual or convective Reynolds number, $\mathrm{Re}=U_{o} A / \nu$, can be expressed as $\mathrm{Re}=\mathrm{We}^{1 / 2} / C$.

(6) The electric number $\chi=A \varepsilon_{o} E_{o}^{2} / \gamma$, also known as Taylor number or electrical Bond number, comparing the electric pressure with the capillary pressure. It can be conceived as the ratio between the capillary time scale and a time scale obtained by geometric averaging of the viscous diffusion time $t_{v}$ and the shear or electrohydrodynamic time scale $t_{s}=\mu /\left(\varepsilon_{o} E_{o}^{2}\right)$, i.e., $\quad \chi \sim t_{c}^{2} /\left(t_{v} t_{s}\right)$ $\sim C t_{c} / t_{s}$. Saville ${ }^{9}$ introduced a Reynolds number based on the electrohydrodynamic velocity $u_{s}=A / t_{s}$; it can be written as $\operatorname{Re}_{s}=\rho \varepsilon_{o} A^{2} E_{o}^{2} / \mu^{2}=\chi / C^{2}$.

(7) The liquid's relative permittivity $\beta=\varepsilon_{i} / \varepsilon_{o}$. The ambient fluid is supposed to have vacuum permittivity.

(8) The relaxation parameter $\alpha=\left[\rho A^{3} K^{2} /\left(\gamma \varepsilon_{i}^{2}\right)\right]^{1 / 2}=t_{c} / t_{e}$. It coincides with the ratio of the electrical relaxation to the capillary time (generally, the shortest hydrodynamic time of the process). The combination $\alpha \beta$ will be shown later to describe the relative importance of conductive versus convective charge transport. The perfectconductor limit is recovered when $\alpha \beta \gg 1$ (generally simplified to $\alpha \gg 1$ by taking into account customary $\beta$ values): this limit implies that "relaxation is quicker than deformation," 32 so that the jet remains isopotential at any time, and its internal electric field is zero. In the opposite situation $\alpha \beta \ll 1$, charges are glued to the interface ("honey-bubble" limit), and are therefore passively convected and stretched with it.

Each perturbative situation is therefore characterized by a set of eight free parameters: $\alpha, \beta, C, \chi, b, \mathrm{We}, \mathrm{We}_{\infty}$, and $\lambda$, whose influence will be explored next. It is important to note that our hypothesis about the electric charge being relaxed to the jet surface does not necessarily imply $\alpha \gg 1$. Indeed, provided that the jet is long enough, the relaxation process can be considered to have taken place at an earlier location. Therefore, the influence of $\alpha$ when it is of $O(1)$ or even smaller can be explored under the assumption that all 
TABLE I. Estimated values in electrospray jets: diameter $A$, relaxation parameter $\alpha$, and Ohnesorge number $C$; experimental data and scaling law from Gañán-Calvo (Ref. 36) and Gañán-Calvo et al. (Ref. 38).

\begin{tabular}{|c|c|c|c|c|c|c|c|}
\hline Liquid & $d_{o}(\mu \mathrm{m})$ & $Q_{o}(\mathrm{ml} / \mathrm{min})$ & $\beta$ & $Q^{\prime} Q_{o}$ & $A(\mu \mathrm{m})$ & $\alpha$ & $C$ \\
\hline \multirow{2}{*}{ Dodecanol $^{\mathrm{a}}$} & \multirow{2}{*}{1.67} & \multirow{2}{*}{2.34} & \multirow{2}{*}{6.5} & 2 & 2.36 & 0.08 & 1.70 \\
\hline & & & & 30 & 9.16 & 0.62 & 0.86 \\
\hline \multirow{2}{*}{ 1-Octanol ${ }^{\mathrm{a}}$} & \multirow{2}{*}{1.52} & \multirow{2}{*}{1.88} & \multirow{2}{*}{10} & 2 & 2.15 & 0.05 & 1.25 \\
\hline & & & & 30 & 8.33 & 0.41 & 0.63 \\
\hline \multirow{2}{*}{ Propylenglycol $^{\mathrm{a}}$} & \multirow{2}{*}{1.24} & \multirow{2}{*}{1.54} & \multirow{2}{*}{31.2} & 2 & 1.76 & 0.02 & 5.18 \\
\hline & & & & 30 & 6.82 & 0.13 & 2.63 \\
\hline \multirow{2}{*}{ Dioxane $^{b}$} & \multirow{2}{*}{5.82} & \multirow{2}{*}{14.5} & \multirow{2}{*}{2.5} & 2 & 4.62 & 0.09 & 0.11 \\
\hline & & & & 30 & 20.72 & 0.86 & 0.05 \\
\hline \multirow{2}{*}{ Heptane $^{b}$} & \multirow{2}{*}{7.43} & \multirow{2}{*}{21.2} & \multirow{2}{*}{1.9} & 2 & 5.89 & 0.12 & 0.04 \\
\hline & & & & 30 & 26.46 & 1.13 & 0.02 \\
\hline
\end{tabular}

${ }^{\mathrm{a}}$ Reference 36

${ }^{\mathrm{b}}$ Reference 38 .

charges are superficial. This is a realistic situation, frequently observed in experimental work.

Electrospray can be used to illustrate the above. This is an apt technique for the generation of electrified capillary microjets nanojets and sprays. It gives rise to a great variety of jet configurations and atomization modes; among them, the cone-jet mode is preferred because of its steadiness and spray characteristics. ${ }^{33}$ Cone-jet operation can be attained with a wide range of liquids ${ }^{34}$ exhibiting enormous diversity in the relevant physical constants (conductivity $K$, surface tension $\sigma$, density $\rho$, relative permittivity $\beta$, and viscosity $\mu$ ). Conductivity and, to a lesser extent, viscosity coefficients, may exhibit radically different orders of magnitude. Cone jets have been obtained with toluene $\left(K=10^{-11} \mathrm{~S} / \mathrm{m}\right)$, intensely acid water solutions $(K=1 \mathrm{~S} / \mathrm{m})$, hexane $(\mu=3$ $\left.\times 10^{-4} \mathrm{~Pa} \mathrm{~s}\right)$ or glycerol ( $\left.\mu=1.3 \mathrm{~Pa} \mathrm{~s}\right)$.

Experimental and theoretical investigations s, $31,35-37^{2}$ have led to the introduction of the following scaling magnitudes:

$$
Q_{o}=\frac{\gamma \varepsilon_{o}}{\rho K}, \quad I_{o}=\left(\frac{\varepsilon_{o} \gamma^{2}}{\rho}\right)^{1 / 2}, \quad d_{o}=\left(\frac{\varepsilon_{o}^{2} \gamma}{\pi^{2} \rho K^{2}}\right)^{1 / 3} .
$$

The above magnitudes set the scale for the injected flow rate $Q$, the electric current transported by the jet $I$, and the jet diameter $A$. With their help, a universal ratio is established: $I / I_{o} \sim\left(Q / Q_{o}\right)^{1 / 2}$ and $A / d_{o} \sim\left(Q / Q_{o}\right)^{1 / 2}$. Provided secondorder effects such as the needle geometry are ignored, the above scaling is accurate in a wide range of situations involving diverse liquids and geometries.

On the basis of electrospray scaling, $\alpha$ and $C$ can be estimated in typical liquid jets issuing from a cone-jet source. Table I shows $\alpha$ and $C$ values drawing on experimental data from Gañán-Calvo, ${ }^{36}$ different organic liquids are used. Some additional data from Gañán-Calvo et al. ${ }^{38}$ were obtained by electrospraying low-viscosity liquids (heptane, dioxane, as well as dodecanol). As can be gathered from the new data, Ohnesorge numbers well below unity can be found by electrospraying nonpolar liquids such as toluene, cyclohexane, or heptane, characterized by their low conductivity and viscosity. Such low Ohnesorge numbers are called to play an increasing role in multiple technological applications involving organic liquids.

These results indicate that low $\alpha$ and $\beta$ are met in realistic experimental explorations. Electrical polarization, the key to dielectric energy storage, is the result of a wide variety of processes, including distortion or reorientation of molecules and orbitals as well as electrochemical reactions. Low values of $\beta$, implying weak polarizability, are frequently observed in connection with organic or long-chain molecules, particularly when water is absent from the composition of the liquid. Ionic charge transport is reduced in low- $\beta$ liquids because of their reluctance to dissolve ions, so that low conductivities are frequent in such cases. Accordingly, the routine assumption that jets are perfect conductors cannot be made without risk. In addition, very low Ohnesorge numbers are frequently observed in low-viscosity jets.

In particular, López-Herrera and Gañán-Calvo ${ }^{19}$ carried out a set of experiments with precisely the same electric field and configuration proposed here. In the above paper, the electrification of the jet tries to achieve the condition of zerotangential stress. Experiments leading to breakup were carried out and compared with a theoretical model assuming perfect-conductor behavior. Three mixtures of water and glycerine were used in the study. Agreement between experiments and theory was in general good, a logical consequence of the fact that all the jets tested were water solutions; and a small amount of water is all that is needed for the permittivity to become high (so that $\alpha \beta \gg 1$, close to the perfectconductor limit). Should the experimental range be expanded to include purely organic liquids, the perfect-conductor hypothesis would no longer hold. The need for a stability theory of imperfect conductors follows from such considerations.

\section{B. Equations}

Under the conditions and assumptions described above, the nondimensional, cylindrical coordinates equations in a reference frame moving with the jet velocity $U_{o}$ are 


$$
\begin{aligned}
& \frac{(r U)_{r}}{r}+W_{z}=0, \\
& U_{t}+U U_{r}+W U_{z}=-P_{r}+C\left(U_{z z}-W_{z r}\right),
\end{aligned}
$$

and

$$
W_{t}+U W_{r}+W W_{z}=-P_{z}+C\left(W_{r r}+\frac{W_{r}}{r}+W_{z z}\right),
$$

where $r$ and $z$ are the radial and the axial coordinates, $t$ is the time, $U$ and $W$ are the radial and axial velocity, and $P$ is the pressure. Subscripts denote partial derivatives.

In the chosen frame, neglecting the gas viscosity, we can approximate the axial speed of the gas as $U_{\infty}-U_{o}$, which in our scaling amounts to $\mathrm{We}_{\infty}^{1 / 2}-\mathrm{We}^{1 / 2}$. Therefore, the equations for the gas phase are

$$
\begin{aligned}
& \frac{\left(r U_{g}\right)_{r}}{r}+\left(W_{g}\right)_{z}=0, \\
& \bar{\rho}\left[\left(U_{g}\right)_{t}+\left(\mathrm{We}_{\infty}^{1 / 2}-\mathrm{We}^{1 / 2}\right)\left(U_{g}\right)_{z}\right]=-\left(P_{g}\right)_{r},
\end{aligned}
$$

and

$$
\bar{\rho}\left[\left(W_{g}\right)_{t}+\left(\mathrm{We}_{\infty}^{1 / 2}-\mathrm{We}^{1 / 2}\right)\left(W_{g}\right)_{z}\right]=-\left(P_{g}\right)_{z} .
$$

The hydrodynamic variables are subject to periodicity conditions in the axial direction

$$
\begin{aligned}
& \left.F_{z}\right|_{z=0}=\left.F_{z}\right|_{z=\lambda}=0, \\
& \left.W\right|_{z=0}=\left.W\right|_{z=\lambda}=0, \\
& \left.U_{z}\right|_{z=0}=\left.U_{z}\right|_{z=\lambda}=0,
\end{aligned}
$$

where $r=F(z, t)$ is the interface equation. In addition, the regularity conditions at the axis demand

$$
\left.U\right|_{r=0}=\left.W_{r}\right|_{r=0}=\left.P_{r}\right|_{r=0}=0 .
$$

The electric problem is modeled by the Laplace equation for both the inner potential $\phi^{i}$ and the outer potential $\phi^{o}$

$$
\nabla^{2} \phi^{i}=\nabla^{2} \phi^{o}=0,
$$

subject to the radial boundary conditions

$$
\left.\phi^{o}\right|_{r=b}=0
$$

and

$$
\left.\phi_{r}^{i}\right|_{r=0}=0,
$$

and periodicity in the axial direction

$$
\left.\phi_{z}^{i}(r, z)\right|_{z=0, \lambda}=\left.\phi_{z}^{o}(r, z)\right|_{z=0, \lambda}=0
$$

for both the inner and outer potentials.

At the interface $r=F(z, t)$, the electrical boundary conditions are

$$
\left.\left(E_{n}^{o}-\beta E_{n}^{i}\right)\right|_{r=F(z, t)}=\sigma_{e}
$$

and

$$
\left.\phi^{o}\right|_{r=F(z, t)}=\left.\phi^{i}\right|_{r=F(z, t)},
$$

where $\sigma_{e}$ is the surface charge density and $E_{n}^{o}$ and $E_{n}^{i}$ are the normal component of the electric field for the outer and inner domain, respectively. The normal and tangential components of the electric field at the interface can be written in terms of the potential as

$$
E_{n}^{o, i}=\left.\frac{1}{\left(1+F_{z}^{2}\right)^{1 / 2}}\left(-\phi_{r}^{o, i}+F_{z} \phi_{z}^{o, i}\right)\right|_{r=F(z, t)}
$$

and

$$
E_{t}=\left.\frac{1}{\left(1+F_{z}^{2}\right)^{1 / 2}}\left(-F_{z} \phi_{r}^{o}-\phi_{z}^{o}\right)\right|_{r=F(z, t)} .
$$

In addition, the kinematic conditions for the liquid and for the gas, the continuity equation of the surface charge density, and the stress balance in the normal and tangential direction read

$$
\left.\left(F_{t}-U+F_{z} W\right)\right|_{r=F(z, t)}=0,
$$

$$
\left.\left\{U_{g}-U+\left(\mathrm{We}^{1 / 2}-\mathrm{We}_{\infty}^{1 / 2}\right) F_{z}\right\}\right|_{r=F(z, t)}=0,
$$

$$
\begin{aligned}
\left(\sigma_{e}\right)_{t} & +\frac{F_{z} U+W}{1+F_{z}^{2}}\left(\sigma_{e}\right)_{z}-\alpha \beta E_{n}^{i} \\
& -\left.\frac{\sigma_{e}}{1+F_{z}^{2}}\left\{U_{r}+F_{z}^{2} W_{z}-F_{z}\left(U_{z}+W_{r}\right)\right\}\right|_{r=F(z, t)}=0,
\end{aligned}
$$

$$
\begin{aligned}
& \left.\left(P-P_{g}\right)\right|_{r=F(z, t)}-p_{\mathrm{ce}} \\
& \quad-\left.\frac{2 C}{1+F_{z}^{2}}\left\{U_{r}+F_{z}^{2} W_{z}-F_{z}\left(W_{r}+U_{z}\right)\right\}\right|_{r=F(z, t)}=0,
\end{aligned}
$$

and

$$
\begin{aligned}
& \left.\frac{C}{1+F_{z}^{2}}\left\{2 F_{z}\left(U_{r}-W_{z}\right)+\left(1-F_{z}^{2}\right)\left(W_{r}+U_{z}\right)\right\}\right|_{r=F(z, t)} \\
& \quad-\chi \sigma_{e} E_{t}=0 .
\end{aligned}
$$

The capillary-electric pressure term is defined as

$$
\begin{aligned}
p_{\mathrm{ce}}= & \frac{1}{\left(1+F_{z}^{2}\right)^{1 / 2}}\left(\frac{1}{F}-\frac{F_{z z}}{1+F_{z}^{2}}\right) \\
& -\frac{\chi}{2}\left[\left(E_{n}^{o}\right)^{2}-\beta\left(E_{n}^{i}\right)^{2}+(\beta-1)\left(E_{t}\right)^{2}\right] .
\end{aligned}
$$

Note that the electrification number $\chi$ weighs the relative importance of the capillary pressure jump (destabilizing for long wavelengths) against the normal component of 
Maxwell's stress, thereby creating the appearance of an altered surface tension. It will be shown later that provided $\chi$ $>1$ and $b>2.71828$, a wavelength range emerges in the vicinity of $k=0$ where axisymmetric disturbances are stable.

In our linear analysis, initial conditions are only used to define the unperturbed solution, as will be done in Eq. (27).

\section{LINEAR ANALYSIS: 3D APPROACH}

\section{A. Perturbed equations and boundary conditions}

In this section we describe the linear analysis of the problem considered. A similar approach has been adopted by Mestel, ${ }^{39,40}$ who analyzed the linear dynamics of charged jets forced by an axial electric field in the quasi-inviscid and highly viscous limits. In both works the jet is charged and subject to an external uniform axial electric field, the electrical relaxation time being a free parameter. Gamero-Castaño and Hruby $^{30}$ studied filaments emitted by cone jets assuming small wave axisymmetric disturbances. Their results are based on the perfect-conductor simplification $\left(E^{i}=0, E_{t}=0\right)$, an approximation implicitly requiring that $\alpha \beta \rightarrow \infty$; therefore, the application of their results to the honey-bubble limit (charge-convection dominant, i.e., $\alpha=0$ ) demands special caution. Hartman et $a l .{ }^{41}$ studied small wave disturbances (both axisymmetric and helical modes) in a jet issuing from a nozzle cone with the help of a lowest-order 1D model for the slice velocity. Their analysis is restricted to the perfectconductor limit. On the other hand, González et al. ${ }^{7}$ provided a thorough stability analysis of conducting jets under ac radial electric fields. A brief description of the dc case is made drawing on previous work by García. ${ }^{6}$ In their paper, González et al. ${ }^{7}$ take into account surrounding gas dynamics effects using a semiempirical model. Azimuthal perturbation modes (nonaxisymmetric fluctuations) are included in their description, and modal competition is shown to take place under the stimulus of the imposed ac frequency. However, their equations are restricted to the perfect-conductor limit. In the present paper, as indicated in the Introduction, only axisymmetric modes will be considered, a hypothesis generally satisfactory when only dc forcing is considered. Most research on the subject agrees in considering nonaxisymmetric modes as evanescent, i.e., either damped or displaying slower growth as axisymmetric disturbances (see for instance Lin and $\mathrm{Webb}^{42}$ ). Our study concentrates on the influence of arbitrary viscosity, permittivity $\beta$, and conductivity $K$ in the presence of a dc radial electric field. The interplay of these parameters is shown to be a key factor in the emergence of an interfacial boundary layer. In addition, the effect of electrification $\chi$ and electrode distance $b$ is discussed.

The temporal linear analysis looks out for solutions in the form of a small sinusoidal spatial perturbation over the static or basic solution. We take the perfect cylinder to be our static reference; therefore, solutions as follows are examined:

$$
\left(\begin{array}{c}
U(z, r, t) \\
W(z, r, t) \\
P(z, r, t) \\
P_{g}(z, r, t) \\
\phi^{o}(z, r, t) \\
\phi^{i}(z, r, t) \\
\sigma_{e}(z, t) \\
F(z, t)
\end{array}\right)=\left(\begin{array}{c}
0 \\
0 \\
1-\chi / 2 \\
P_{\infty} \\
\ln (b / r) \\
\ln (b) \\
1 \\
1
\end{array}\right)+\left(\begin{array}{c}
\tilde{u}(r, z, t) \\
\widetilde{w}(r, z, t) \\
\tilde{p}(r, z, t) \\
\tilde{p}_{g}(r, z, t) \\
\widetilde{\phi}^{o}(r, z, t) \\
\widetilde{\phi}^{i}(r, z, t) \\
\widetilde{\sigma}_{e}(z, t) \\
\tilde{f}(z, t)
\end{array}\right),
$$

and the perturbation is written as

$$
\left.\left(\begin{array}{c}
\widetilde{u}(r, z, t) \\
\widetilde{w}(r, z, t) \\
\tilde{p}(r, z, t) \\
\widetilde{p}_{g}(r, z, t) \\
\widetilde{\phi}^{o}(r, z, t) \\
\widetilde{\phi}^{i}(r, z, t) \\
\widetilde{\sigma}_{e}(z, t) \\
\tilde{f}(z, t)
\end{array}\right)=\operatorname{Re}\left[\begin{array}{c}
\hat{u}(r) \\
\hat{w}(r) \\
\hat{p}(r) \\
\hat{p}_{g}(r) \\
\hat{\phi}^{o}(r) \\
\hat{\phi}^{i}(r) \\
\hat{\sigma}_{e} \\
\hat{f}
\end{array}\right) \zeta\right],
$$

where $\zeta$ is equal to $e^{\Omega t+i k z}, k$ being the nondimensional wavenumber $\pi / \lambda$ ( $\lambda$ is the half wavelength), and $\Omega=\sigma+\omega i$ is the complex eigenvalue involving the growth rate $\sigma$ and the oscillation frequency $\omega$. Hatted variables denote small amplitudes. Neglecting nonlinear terms of the Navier-Stokes equations we obtain

$$
\boldsymbol{\nabla} \cdot \widetilde{\mathbf{v}}=0
$$

and

$$
\widetilde{\mathbf{v}}_{t}=-\nabla \widetilde{p}+C \nabla^{2} \widetilde{\mathbf{v}}
$$

A separate equation for the pressure can be obtained by applying the divergence operator to (30) and using (29); the same holds for the gas pressure:

$$
\nabla^{2} \widetilde{p}=0, \quad \nabla^{2} \widetilde{p}_{g}=0 .
$$

On the other hand, taking the Laplacian of (30) leads to

$$
\nabla^{2}\left(\nabla^{2}-\frac{1}{C} \frac{\partial}{\partial t}\right) \tilde{\mathbf{v}}=0
$$

Due to the linear character of the operators in the latter equation we can split the velocity into two terms $\widetilde{\mathbf{v}}=\widetilde{\mathbf{v}}_{v}+\widetilde{\mathbf{v}}_{n v}$, where $\widetilde{\mathbf{v}}_{n v}$ is the inviscid contribution and $\widetilde{\mathbf{v}}_{v}$ the viscous contribution; the following equations are satisfied:

$$
\nabla^{2} \widetilde{\mathbf{v}}_{n v}=0, \quad\left(\nabla^{2}-\frac{1}{C} \frac{\partial}{\partial t}\right) \widetilde{\mathbf{v}}_{v}=0, \quad \widetilde{\mathbf{v}}_{n v_{t}}=-\nabla \widetilde{p} .
$$

The perturbations comply with the regularity condition at the axis $\left.\widetilde{p}(r, z, t)\right|_{r=0}$ and $\left.\widetilde{w}(r, z, t)\right|_{r=0}$ finite and $\left.\widetilde{u}(r, z, t)\right|_{r=0}=0$. By Taylor expansion around $r=1$ at the interface, the kinematic conditions and the stress balance yield

$$
\left.\widetilde{u}\right|_{r=1}=\tilde{f}_{t},
$$




$$
\begin{aligned}
& \left.\left(\widetilde{u}-\widetilde{u}_{g}\right)\right|_{r=1}=i k\left(-\mathrm{We}_{\infty}^{1 / 2}+\mathrm{We}^{1 / 2}\right) \tilde{f}, \\
& \left.\left(\widetilde{p}-\widetilde{p}_{g}\right)\right|_{r=1}=-\tilde{f}-\widetilde{f}_{z z}+\left.2 C \widetilde{u}_{r}\right|_{r=1}+\left.\chi\left(\tilde{f}+\widetilde{\phi}_{r}^{o}\right)\right|_{r=1},
\end{aligned}
$$

and

$$
\left.C\left(\widetilde{u}_{z}+\widetilde{w}_{r}\right)\right|_{r=1}+\left.\chi \widetilde{\phi}_{z}^{o}\right|_{r=1}=0 .
$$

The outer and the inner electrical potential perturbations satisfy the Laplace equation

$$
\nabla^{2} \widetilde{\phi}^{o, i}=0
$$

with regularity conditions,

$$
\left.\widetilde{\phi}^{i}(r, z, t)\right|_{r=0} \text { finite, }\left.\quad \widetilde{\phi}^{o}(r, z, t)\right|_{r=b}=0 .
$$

At the interface the inner and the outer potential are coupled through the normal electric field jump and the continuity of the potential

$$
\left.\left(-\widetilde{\phi}_{r}^{o}+\beta \widetilde{\phi}_{r}^{i}\right)\right|_{r=1}=\widetilde{\sigma}_{e}
$$

and

$$
\left.\widetilde{\phi}^{o}\right|_{r=1}=\left.\widetilde{\phi}^{i}\right|_{r=1}
$$

together with the continuity equation for the charge surface density

$$
\left(\widetilde{\sigma}_{e}\right)_{t}-\left.\widetilde{u}_{r}\right|_{r=1}=-\left.\alpha \beta \widetilde{\phi}_{r}^{i}\right|_{r=1} .
$$

Substituting the solution (27) in the bulk equations (31)-(33) and (38) we obtain the ODE system which governs the amplitudes of the variables

$$
\begin{aligned}
& \Psi^{\prime \prime}+\frac{\Psi^{\prime}}{r}-k^{2} \Psi=0, \\
& \hat{w}_{v}^{\prime \prime}+\frac{\hat{w}_{v}^{\prime}}{r}-k_{v}^{2} \hat{w}_{v}=0, \\
& \hat{u}_{n v}^{\prime \prime}+\frac{\hat{u}_{n v}^{\prime}}{r}-\left(k^{2}+\frac{1}{r^{2}}\right) \hat{u}_{n v}=0, \\
& \hat{u}_{v}^{\prime \prime}+\frac{\hat{u}_{v}^{\prime}}{r}-\left(k_{v}^{2}+\frac{1}{r^{2}}\right) \hat{u}_{v}=0,
\end{aligned}
$$

where $\Psi$ stands for $\hat{p}, \hat{p}_{g}, \hat{w}_{n v}$, and $\hat{\phi}^{o, i}$. The primes denote differentiation with respect to $r$ and $k_{v}$ is defined as

$$
k_{v}^{2}=k^{2}+\frac{\Omega}{C} .
$$

The additive term $\Omega / C \sim \bar{\Omega} A^{2} / \nu \sim t_{v} / t_{d}$ is occasionally identified as a Reynolds number based on the problem's unsteady character, $\bar{\Omega}$ being the physical magnitude of $\Omega{ }^{39}$ it can also be viewed as the ratio between the viscous diffusion time scale $t_{v}$ and the disturbance time scale $t_{d}$. In addition, the continuity and momentum equations read

$$
\hat{u}^{\prime}+\frac{\hat{u}}{r}+i k \hat{w}=0, \quad \hat{p}^{\prime}=-\Omega \hat{u}_{n v}, \quad i k \hat{p}+\Omega \hat{w}_{n v}=0 .
$$

The gas momentum equation in the radial direction is

$$
\bar{\rho}\left[\Omega+i k\left(\mathrm{We}_{\infty}^{1 / 2}-\mathrm{We}^{1 / 2}\right)\right] \hat{u}_{g}=-\hat{p}_{g}^{\prime} .
$$

The boundary conditions closing the problem are

$$
\begin{aligned}
& \hat{u}(0)=0, \hat{w}(0), \hat{p}(0) \text { and } \hat{\phi}^{i}(0) \text { finite, } \hat{p}_{g}(\infty)=0 ; \\
& \hat{u}(1)=\Omega \hat{f}, \hat{u}_{g}(1)=\hat{u}(1)+i k\left(\mathrm{We}_{\infty}^{1 / 2}-\mathrm{We}^{1 / 2}\right) \hat{f}, \\
& \hat{p}(1)-\hat{p}_{g}(1)=\left(-1+k^{2}\right) \hat{f}+2 C \hat{u}^{\prime}(1)+\chi\left[f+\left(\hat{\phi}^{o}\right)^{\prime}(1)\right],
\end{aligned}
$$

$$
\begin{aligned}
& C\left[i k \hat{u}(1)+\hat{w}^{\prime}(1)\right]+\chi i k \hat{\phi}^{i}(1)=0, \\
& -\hat{f}+\hat{\phi}^{o}(1)=\hat{\phi}^{i}(1), \\
& -\hat{f}-\left(\hat{\phi}^{o}\right)^{\prime}(1)+\beta\left(\hat{\phi}^{i}\right)^{\prime}(1)=\hat{\sigma}_{e},
\end{aligned}
$$

$$
\Omega \hat{\sigma}_{e}-\hat{u}^{\prime}(1)=-\alpha \beta\left(\hat{\phi}^{i}\right)^{\prime}(1),
$$

$$
\hat{\phi}^{o}(b)=0 .
$$

\section{B. The dispersion equation}

The general solution of the ODE set (43)-(48) consists of a combination of modified Bessel functions. Recalling (50) and (57), the solution reads

$$
\begin{aligned}
& \hat{p}(r)=-\mathcal{A} \frac{\Omega I_{o}(k r)}{k I_{1}(k)}, \quad \hat{p}_{g}(r)=\mathcal{A}_{g} \frac{K_{o}(k r)}{k K_{1}(k)}, \\
& \hat{u}(r)=\hat{u}_{n v}(k r)+\hat{u}_{v}(k r)=\mathcal{A} \frac{I_{1}(k r)}{I_{1}(k)}-\mathcal{B} \frac{I_{1}\left(k_{v} r\right)}{I_{1}\left(k_{v}\right)},
\end{aligned}
$$

and

$$
\hat{w}(r)=\hat{w}_{n v}(k r)+\hat{w}_{v}(k r)=\mathcal{A} i \frac{I_{o}(k r)}{I_{1}(k)}-\mathcal{B} i \frac{k_{v} I_{o}\left(k_{v} r\right)}{k I_{1}\left(k_{v}\right)}
$$

for the hydrodynamic variables, and

$$
\hat{\phi}^{o}(r)=\frac{\mathcal{A}_{e}\left[K_{o}(k b) I_{o}(k r)-I_{o}(k b) K_{o}(k r)\right]}{k\left[K_{o}(k b) I_{1}(k)+I_{o}(k b) K_{1}(k)\right]}
$$

and

$$
\hat{\phi}^{i}(r)=\mathcal{B}_{e} \frac{I_{o}(k r)}{k I_{1}(k)}
$$

for the outer and inner electrical potential, where $\mathcal{A}, \mathcal{A}_{g}, \mathcal{B}, \mathcal{A}_{e}$, and $\mathcal{B}_{e}$ are real constants. Note that, under adequate scaling, the radial profiles of the pressure and the electric potential are identical. The substitution of the previous solutions in the boundary conditions at the interface (50)-(57) and the elimination of the constants $\mathcal{A}, \mathcal{A}_{g}, \mathcal{B}, \mathcal{A}_{e}$, and $\mathcal{B}_{e}$ leads to the dispersion relation 


$$
\begin{aligned}
\mathcal{D}= & \Omega^{2} \xi(k)+2 C \Omega\left[2 k^{2} \xi(k)-1\right]+4 k^{2} C^{2}\left[k^{2} \xi(k)\right. \\
& \left.-k_{v}^{2} \xi\left(k_{v}\right)\right]+\frac{2 \Xi(k) C}{\Omega} k^{2}[2+G(k)]\left[k^{2} \xi(k)-k_{v}^{2} \xi\left(k_{v}\right)\right] \\
& +\Xi(k)\left(2 k^{2} \xi(k)+k^{2} \xi(k) k_{v}^{2} \xi\left(k_{v}\right) G(k)+\frac{1}{G(k)}\right) \\
& +\left(1+\frac{\Xi(k) k^{2} G(k)}{\Omega^{2}}\left[k_{v}^{2} \xi\left(k_{v}\right)-k^{2} \xi(k)\right]\right) T(k)=0,
\end{aligned}
$$

where the driving term $T(k)$ sums up all the interfacial forcing (surface tension, aerodynamic effects, electric charging) and is defined as

$$
\begin{aligned}
T(k)= & k^{2}-1+\chi\left(1+\frac{1}{G(k)}\right) \\
& -\bar{\rho}\left[\Omega+i k\left(\mathrm{We}_{\infty}^{1 / 2}-\mathrm{We}^{1 / 2}\right)\right]^{2} \Gamma(k),
\end{aligned}
$$

while the following auxiliary functions are introduced:

$$
\begin{aligned}
& G(k)=\frac{K_{o}(k b) I_{o}(k)-I_{o}(k b) K_{o}(k)}{k\left[K_{o}(k b) I_{1}(k)+I_{o}(k b) K_{1}(k)\right]}, \quad \xi(k)=\frac{I_{o}(k)}{k I_{1}(k)}, \\
& \Xi(k)=\frac{\chi \xi(k)}{E(k)}, \quad E(k)=G(k) \beta\left(1+\frac{\alpha}{\Omega}\right)-\xi(k),
\end{aligned}
$$

and

$$
\Gamma(k)=-\frac{K_{o}(k)}{k K_{1}(k)} .
$$

$\Xi(k)$ is a combined electrification number, barely sensitive to $b$, condensing all the influence of $\alpha$ and $\beta$; its magnitude increase as the conductivity or the permittivity decrease. $\Xi(k)$ becomes zero in the perfect-conductor limit, when either $\alpha, \beta$, or their combination tend to $\infty$. $E(k)$ is always negative. In the limit where $b \gg 1, G(k) \rightarrow \Gamma(k)$. The leading term in $\mathcal{D}$ is $\Omega^{2} \xi(k)$, representing the effect of inertia, i.e., forces caused by the unsteadiness of the disturbance. In lowviscosity jets $(C \ll 1)$, this term becomes dominant and is balanced by the driving term alone. In the opposite limit $(C \gg 1)$, the driving term is balanced by the viscous terms alone (second and third terms in $\mathcal{D}$ ). The calculations leading to the dispersion relation are detailed in the Appendix.

It can be readily verified that $\alpha \beta \rightarrow \infty$ implies that $\mathcal{B}_{e}$ $\rightarrow 0$, so that the inner electric field becomes vanishingly small. Assuming the disturbances are small, it follows as well that the tangential field $E_{t}$ becomes negligible. Therefore, the perfect-conductor case can be recovered by selecting $\alpha \beta \gg 1$.

Our description of the influence of the surrounding gas mirrors the procedure introduced by González et al. $;^{7}$ in their paper they suggest a simple semiempirical correction, based on Sterling and Sleicher, ${ }^{43}$ allowing the description of the gas viscosity influence. Additionally, concerning the validity range of the theory, the work of Lin and Reitz ${ }^{25}$ is cited to justify the parametric range where the above applies: We $>4, \bar{\rho} \mathrm{We}<6.5$. These limits are empirically consistent for unelectrified jets only, but can be used as orientative land-

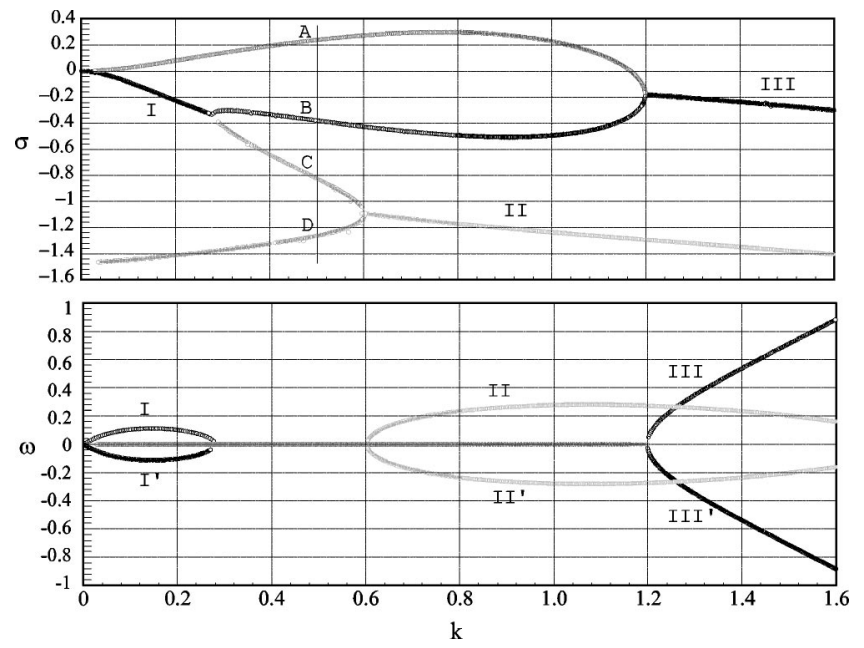

FIG. 2. Solution of the dispersion relation $(\alpha=1, \beta=10, C=0.1$, and $\chi$ $=0.6$ ).

marks in our case. The first restriction is aimed at avoiding absolute instability (dripping mode); it sets a criterion for Rayleigh breakup. The second restriction excludes situations belonging to the second wind-induced breakup regime, where gas shear becomes important. However, the range of validity is not well studied in the case where the outer fluid is flowing co-axially with a speed $U_{\infty}$. In spite of the resulting uncertainty, the dispersion equation (63) is to be trusted whenever dripping or second wind-induced breakup can be excluded.

Figure 2 shows a characteristic stability map; the growth rate $\sigma$ and the oscillation frequency $\omega$ are plotted against the wavenumber $k$. The jet is characterized by an Ohnesorge number $C=0.1$, Taylor number $\chi=0.6$, relative permittivity $\beta=10$, relaxation parameter $\alpha=1.0$, and $\beta=\infty$. Aerodynamic effects are ignored. A single unstable branch is observed, corresponding to values of $k$ ranging from 0 to 1.16703 , associated with aperiodic growth of perturbations. The other branches are stable, some of them being oscillatory stable (I, II, and III). There are four complex solutions associated to each wavenumber. In Fig. 3 the velocity fields and external electric fields (vectorial) are depicted for each one of the solutions; $k=0.5$, a regime where all behaviors are aperiodic ( $\Omega$ is a real number). We observe that (i) the electric field is always symmetrical with respect to the wavelength; (ii) the only unstable mode is essentially capillary (A) while the others are stable. The main stabilizing force in mode B is the surface tension. Modes $\mathrm{C}$ and $\mathrm{D}$ are characterized by strong recirculations of the liquid where the viscous term balances the inertial term, the capillary term being negligible.

An even number of roots are observed. The problem dealt with by Hohman et al. ${ }^{44}$ displays a distinctive difference compared to our problem: the presence of an axial, rather than radial, electric field. The dispersion relation obtained by them is cubic; accordingly, they find three modes; on the other hand, the radial geometry of our electric forcing leads to a fourth-order dispersion relation (four modes).

A salient physical difference between the two problems is that the first one displays antisymmetric electric charges in 

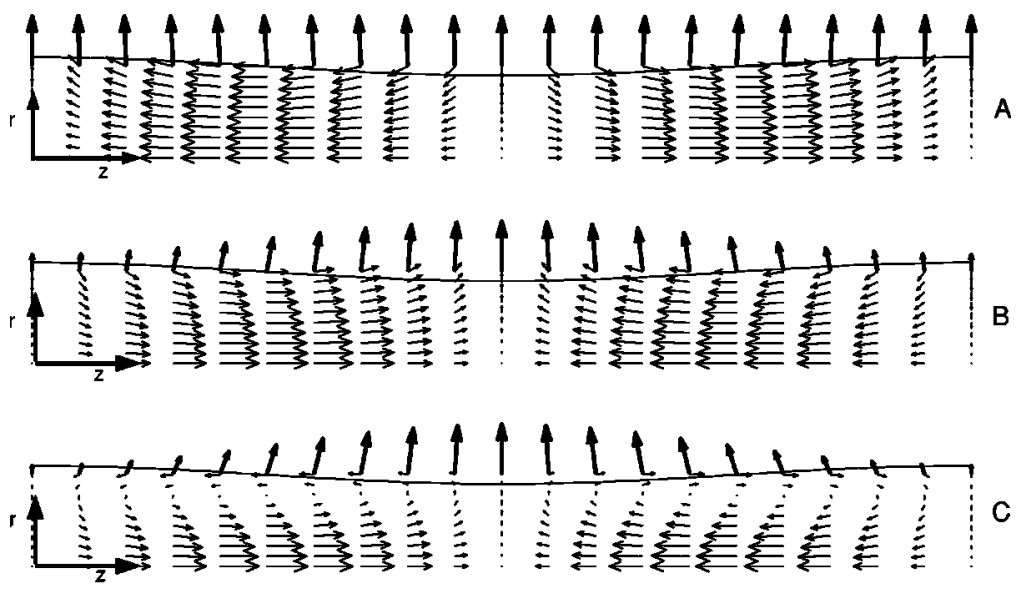

FIG. 3. Jet velocity and interfacial outer field in a wavelength segment for: (A) Capillary unstable mode; (B) capillary stable mode; ( $\mathrm{C}$ and $\mathrm{D})$ recirculating modes $(b \gg 1, k=0.5, \alpha=1, \beta=10, C=0.1$, and $\chi=0.6)$.

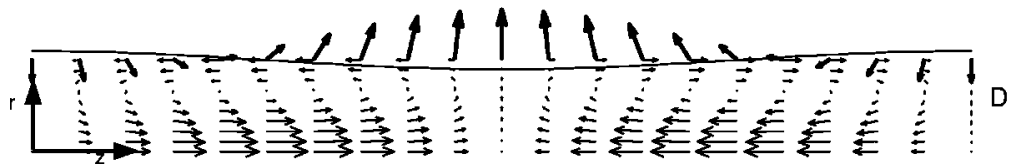

the axial direction along a perturbation wavelength, while ours is symmetric. Their having an odd-order dispersion relation rather than an even-order one may plausibly be a reflection of the antisymmetry inherent to axial forcing. In addition, as illustrated in Fig. 2, the radial electric field induces an additional stable mode (three out of four) while the physical picture drawn by Hohman et al. in the presence of a tangential field clearly hints at the destabilizing role of axial electric forcing. A simplistic but illustrative picture is the following: given a symmetric (cosine-like) perturbation, a radial electric field induces additional dynamical actions which are symmetrical in the axial direction with respect to the perturbation (and these may be stabilizing or destabilizing), while the axial electric field gives rise to antisymmetric forces which are always destabilizing: see Figs. 9 and 11 in Hohman et al. ${ }^{44}$

Aerodynamic effects modify the above map in that nonzero $\bar{\rho}$, We, or $\mathrm{We}_{\infty}$ give rise to an imaginary component of the dispersion equation $\mathcal{D}$. This has an immediate implication: it is impossible for a real $\Omega$ to satisfy the dispersion equation. Therefore, all growth behaviors are modulated, $\operatorname{lm}(\Omega) \equiv \omega \neq 0$, i.e., aperiodic growth (as shown in Fig. 2 for the unstable branch A) cannot exist.

\section{Parametrical patterns of the dispersion equation}

In the uncharged limit $(\chi=0$ in $\mathcal{D})$, the dispersion equation coincides with the expression obtained by Chandrasekhar ${ }^{45}$ after Rayleigh. ${ }^{46}$ Setting the additional constraint $C=0$ leads to the original result for the capillary instability due to Rayleigh. Setiawan and Heister ${ }^{4}$ obtained an expression for $\mathcal{D}$ in the electrified inviscid limit, assuming perfect conductivity and $b=\infty$. Huebner and $\mathrm{Chu}^{3}$ developed a variational approach leading to an expression for $\mathcal{D}$ in the perfect-conductor limit, assuming zero viscosity and arbitrary electrode distance $b$. Artana et al. $^{32}$ included aerodynamic effects and higher stability modes using the same assumptions.

In this section, different growth rate curves are shown. In growth curves, $\sigma=\operatorname{Re}(\Omega)$ is plotted as a function of the wavenumber $k$ in the instability window corresponding to the unstable branch A of Fig. 2. All dispersion curves exhibit maximal growth rate for a particular value of the wavenumber, as well as upper and lower cutoff wavenumbers $\left(k_{1}, k_{2}\right)$, which define our instability window. The fastest growing mode $\left(k_{m}, \sigma_{m}\right)$ is the most relevant quantity in the case of random perturbation (noise). Artana et al., ${ }^{32}$ among others, indicate that the mean diameter of the first droplets produced at the jet's breakup is proportional to the inverse of $k_{m}$ while the intact length of the jet (before detachment of the first droplet) is proportional to the inverse of $\sigma_{m}$.

Provided that the speed difference $U_{o}-U_{\infty}$ is sufficiently large, aerodynamic effects can no longer be neglected. Our exploration shows that as $\bar{\rho}\left(\mathrm{We}_{\infty}^{1 / 2}-\mathrm{We}^{1 / 2}\right)$ increases, the following trend is observed: the instability lobe expands, while $\left(k_{m}, \sigma_{m}\right)$ increase, implying smaller droplet size at breakup. Effects are hardly noticeable when $\bar{\rho}\left(\mathrm{We}_{\infty}^{1 / 2}-\mathrm{We}^{1 / 2}\right)<0.2$. The imaginary part $\omega$ as a function of $k$ is monotonously growing.

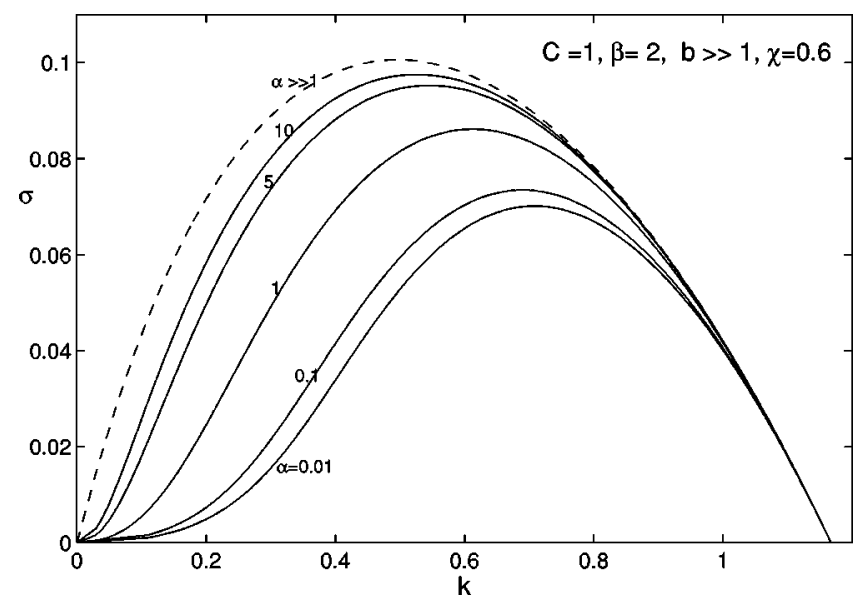

FIG. 4. Growth rate $\sigma$ vs the wavenumber $k$ for a jet with $b \gg 1, \chi=0.6$, $C=1$, and $\beta=2$ and several values of the relaxation parameter $\alpha$. 


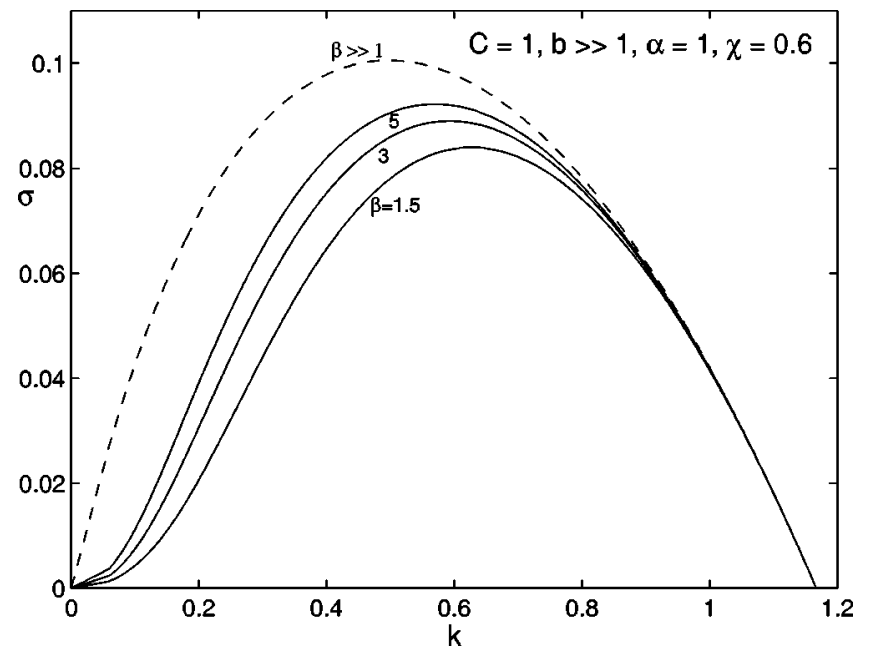

FIG. 5. Growth rate $\sigma$ vs the wavenumber $k$ for a jet with $b \gg 1, \chi=0.6$, $C=1$, and $\alpha=1$ and several values of the relative permittivity $\beta$.

In the ensuing discussion, the effects of the ambient gas will be considered negligible. In the gas-at-rest case, this amounts to the constraint $\bar{\rho} \mathrm{We}<0.2$, marking the onset of the first wind-induced breakup regime.

The influence of the conductivity and permittivity parameters is explored in Figs. 4 and 5, where the growth rate is shown for a jet of moderate viscosity and electrification $(b=\infty, C=1$, and $\chi=0.6)$; Fig. 4 is plotted with $\beta=2$; in Fig. $5, \alpha=1$. It is worth noting that the $\beta$ influence can only be explored provided $\alpha$ is finite. A similar trend is observed for $\alpha$ and $\beta$. Growing conductivity or permittivity results in a decrease of $k_{m}$ and an increase of $\sigma_{m}$, implying larger breakup wavelength and increased instability. Provided that any of these parameters is sufficiently high, the perfectconductor limit is reached.

These results illustrate the influence of $\alpha$ and $\beta$ on the growth rate, and show a clear stabilizing trend associated with imperfect conductivity or permittivity. The stabilization is more evident as the wavelength rises. Particular sensitivity to $\alpha$ and $\beta$ is felt in the long-wavelength range. Imperfect conductivity and permittivity (small enough $\alpha$ or $\beta$ ) gives rise to a slow-growth zone near $k=0$. It is worth noting that poor conductivity becomes irrelevant as the outer electrode gets closer to the jet (lower values of $b$ tend to annihilate the influence of $\beta$ and $\alpha$, bringing results close to the perfectconductor limit). In general, the conducting limit is rapidly reached by increasing the permittivity $\beta$ or the relaxation parameter $\alpha$. In the practice, both parameters tend to be correlated: high permittivity liquids usually exhibit a high electrical conductivity as well, on account of their polarity and their ability to dissolve ionic species. Both parameters have no influence whatsoever on the stability range, which, as will be shown next, is only dependent on $\chi$ and $b$.

Figures 6 and 7 show growth rate curves for different values of the electrification number $\chi$ and the electrode distance $b$. Coincident patterns of influence are observed: increasing the electric field $\chi$ exerts a similar effect as bringing the outer electrode closer. The electrification effect is therefore enhanced by an encroaching electrode. Both parameters have in common a destabilizing influence, reflected in widening instability lobes.

The stability range is very sensitive to electrification. As $\chi$ increases, the instability lobe expands (see, for instance, Baudry et $a l^{5}$ ). Taking the $\Omega \rightarrow 0$ limit in the dispersion equation, under the assumption that $k \neq 0$, leads to an equation for the limits $\left(k_{1}<k<k_{2}\right)$ where a positive real growth rate is found:

$$
T\left(k_{1}\right)=T\left(k_{2}\right)=0,
$$

$T(k)$ being the driving term defined in Eq. (64). The upper and lower bound are plotted in Fig. 8 for different values of $\chi$. In the unelectrified case, the Rayleigh instability limits $\left(k_{1}=0\right.$ and $\left.k_{2}=1\right)$ are recovered: the capillary terms $k^{2}-1$ are, respectively, associated with the transversal curvature (destabilizing) and the longitudinal curvature (stabilizing). Long wavelengths increase the relative importance of the first and thereby cause instability.

Electrification has an ambivalent effect on stability. For large $b$, as discussed by González et al. ${ }^{7}$ (who also provide a
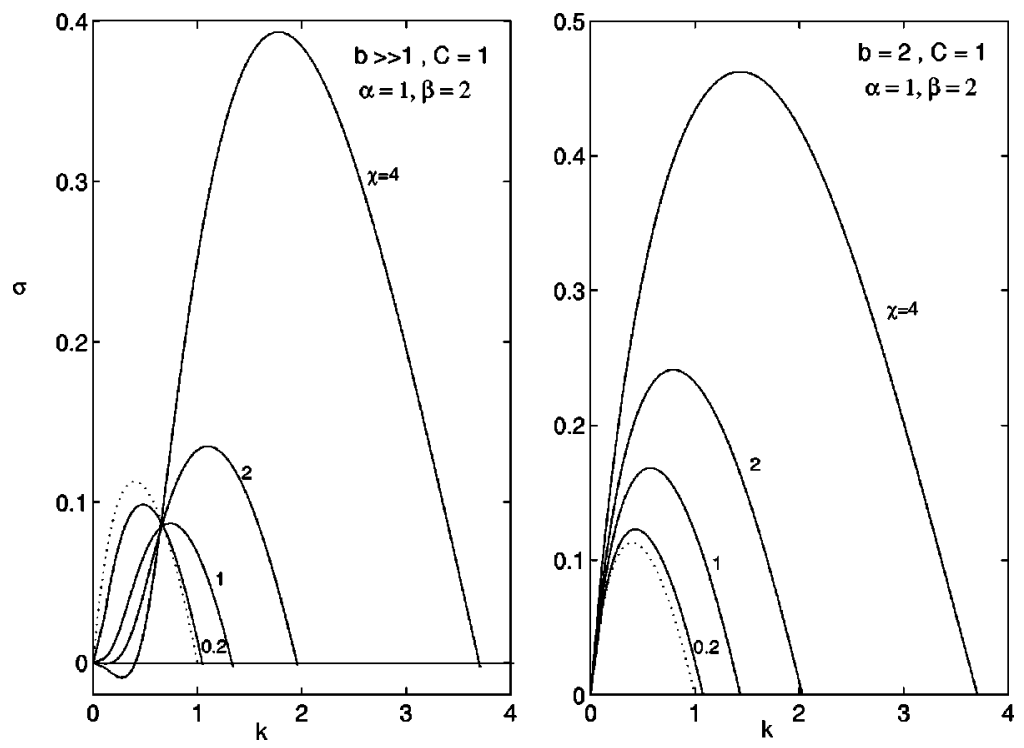

FIG. 6. Growth rate $\sigma$ vs the wavenumber $k$ for a jet with $b \gg 1$ and $b=2$. The other parameters are fixed at $C=1, \alpha=1$, and $\beta=2$. Several values of the electrification number $\chi$ are plotted. 


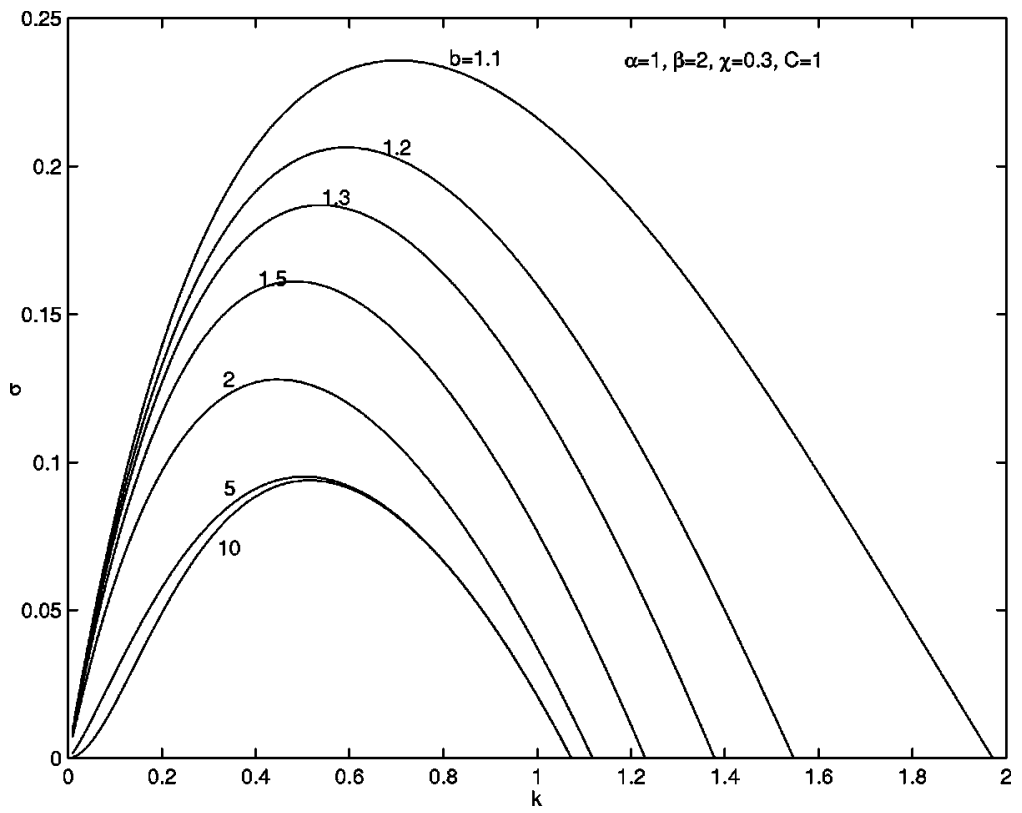

FIG. 7. Growth rate $\sigma$ vs the wavenumber $k$ for a jet with $\alpha=1, \beta=1, C=1, \chi=0.3$, and several values of the grounded electrode distance $b$.

physical interpretation of the dual influence of $\chi$ at the crests and valleys of the disturbance), electric charging of the jet destabilizes short waves while stabilizing long ones. In fact, provided that $\chi$ is larger than 1 and under the condition that

$$
b>\left.G^{-1}\left(-\frac{\chi}{\chi-1}\right)\right|_{k=0}=\exp \left(\frac{\chi}{\chi-1}\right),
$$

the lower bound $k_{1}$ rises above $k=0$, so that a stable region in the range $0<k<k_{1}$ emerges (see Fig. 6, $b \gg 1$; and Fig. 8, where the lower and upper cutoff bounds are plotted as a function of $b$ for diverse values of $\chi$ ). Nevertheless, $k_{1}$ is bounded, and even in the most favorable case $(b$ and $\chi \rightarrow \infty)$, $k_{1}=\Gamma^{-1}(-1)=0.595$. This boundary, in the large- $b$ limit, separates the range where electrostatic forces are stabilizing, as reported by Setiawan and Heister. ${ }^{4}$
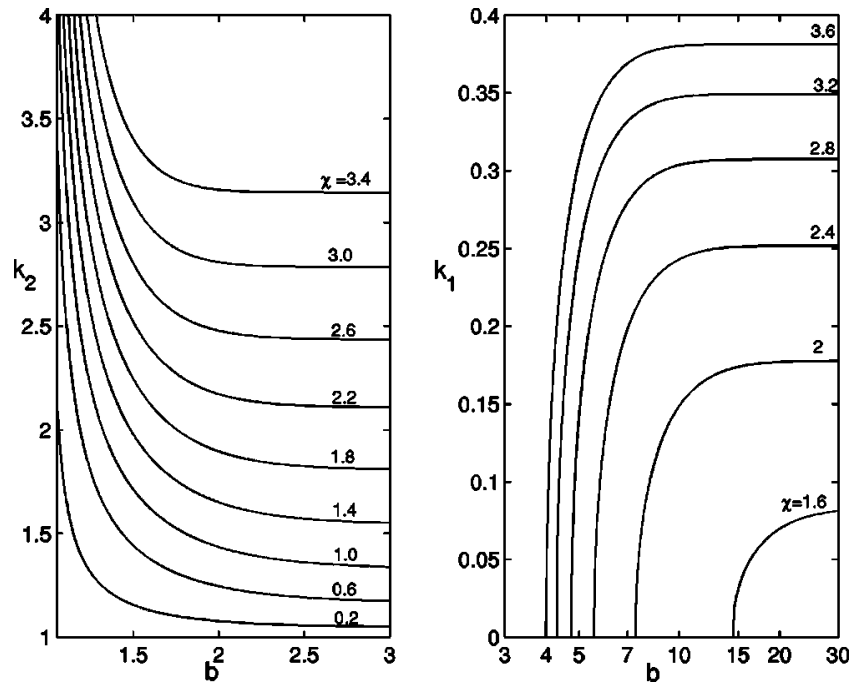

FIG. 8. Lower and upper stability cutoff bounds $k_{1}$ and $k_{2}$ plotted as a function of the ground radial distance $b$ for different values of the electrification.
The above observations are in agreement with recent experimental and numerical studies on perfect-conductor jets ${ }^{17}$ indicating that, in the range of electrification levels and Weber numbers ensuring an axisymmetric breakup, the influence of $\chi$ is merely quantitative. The breakup wavelength and frequency are sensitive to the electrification level, owing to a change in the effective surface tension operated by the electric field. The effects of electrification (and aerodynamic coupling) are summed in the driving term $T(k)$, the only part of the dispersion equation dependent on $\chi, b, U_{\infty}$, and $U_{o}$. However, if the liquid is an imperfect conductor, a tangential electric stress arises which attenuates the growth of perturbations, and may promote longer jets as observed in electrospray experiments using moderate viscosity liquids in the nonwhipping jet regime. ${ }^{12,13,39,40}$

For arbitrary ground location $b$, a threshold value can be defined, $k_{s}=\left.G^{-1}(-1)\right|_{b}$, cutting off the region where electrification is stabilizing $\left(k<k_{s}\right)$ from the region where a destabilizing influence is observed $\left(k>k_{s}\right)$. It is interesting to note that $b$ values lower than the $e$ number $(b<2.71828)$ imply that, whatever the electrification, its effect is always destabilizing, regardless of $k$. Therefore, in the tight-electrode limit $(b \rightarrow 1)$, increasing $\chi$ gives rise to an unanimous increase of the growth rate.

On the other hand, the upper cutoff mode $k_{2}$, also depicted in Fig. 8, is very sensitive to electric charging, particularly when the electrode gets closer to the jet. Indeed, in the tight-electrode limit, the instability range grows asymptotically, so that the entire wavelength range ends up being unstable.

The long-wavelength limit can be explored in the general case by taking the limit of the dispersion equation $\mathcal{D}$ when $k \rightarrow 0$. The positive real root of $\mathcal{D}$ is

$$
\sigma \approx B_{1}(b, \chi) k, \quad B_{1}(b, \chi)=\frac{1}{2}[\chi(3-4 \ln b)+1]
$$

indicating a local linear behavior of the growth rate. This approximation is only correct provided that 


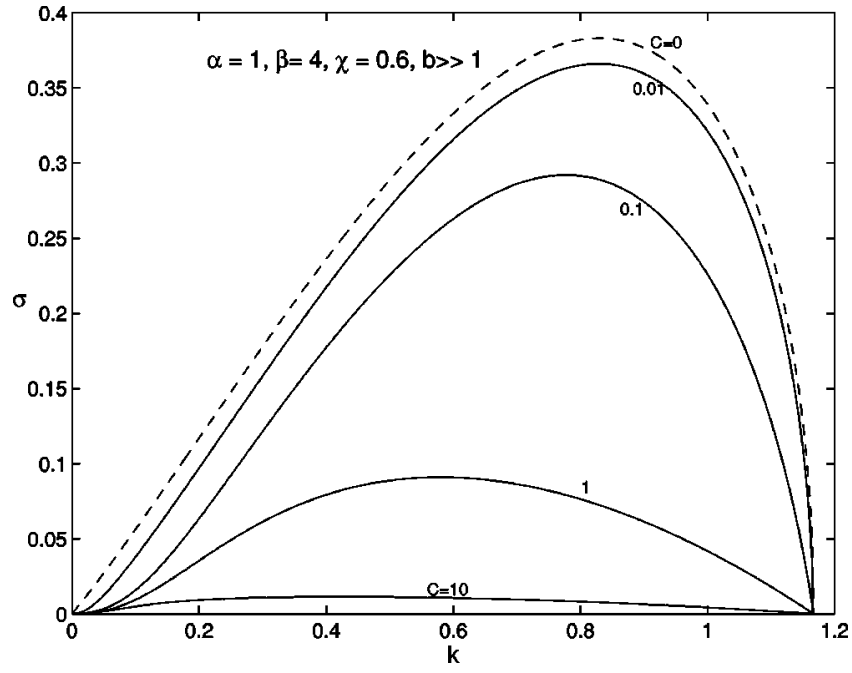

FIG. 9. Growth rate $\sigma$ vs the wavenumber $k$ for a jet with $b \gg 1, \alpha=1, \beta$ $=1, \chi=0.6$, and several values of the Ohnesorge number $C$.

$$
b<\exp \left[\frac{1}{4}\left(3+\frac{1}{\chi}\right)\right]=2.117 e^{1 / 4 \chi}=b_{o}(\chi) .
$$

When $b>b_{o}(\chi)$, the growth rate follows a quadratic trend:

$$
\begin{aligned}
& \sigma \approx B_{2}(b, \chi) k^{2}, \\
& B_{2}(b, \chi)=\frac{(\alpha \beta C+\chi / 2)[(\chi-1) \ln b-\chi]}{2 C(1+3 \chi-4 \chi \ln b)} .
\end{aligned}
$$

A low growth region is observed near $k=0$; the width of this region increases with decreasing $\alpha, \beta$. In order to carry out consistent expansions of the general solution in the $k \ll 1$ limit, it is worth noting that $k_{v}^{2}=k^{2}+\Omega / C$ is of the following order of magnitude:

$$
k_{v}= \begin{cases}O\left(k^{1 / 2}\right) & \text { when } \quad b<b_{o}(\chi) \\ O(k) & \text { when } \quad b>b_{o}(\chi) .\end{cases}
$$

The above does not hold when $C \rightarrow 0$, because the emergence of an interface boundary layer modifies the small- $k$ patterns.

Equations (70) and (72) confirm the existence of a low$k$ stability region $\left[0, k_{1}\right]$ as illustrated by Figs. 6 and 8 . Indeed, for sufficiently large values of $b$ and $\chi$, the local values of $\sigma$ switch from positive to negative.

\section{The influence of viscosity and the boundary layer at the interface in the low-viscosity limit \\ 1. High-viscosity limit}

The influence of the viscosity can be illustrated by comparing growth curves having diverse Ohnesorge numbers (see Fig. 9). As viscosity increases, the most unstable wavelength becomes longer and the growth rate decreases, corresponding to an increasingly uniform and stiff flow regime and therefore increasingly limited deformability. ${ }^{47}$ Viscosity does not modify the stability limits, but it lowers the growth rate of any perturbation. High Ohnesorge numbers are also linked to the addition of surfactants, all of which possess the common property of lowering surface tension when added to liquids in small amounts. ${ }^{48}$

In the high-viscosity limit $\left(C \gg \Omega\right.$, i.e., $t_{v} \ll t_{d}$, where $t_{v}$ is the viscous diffusion time scale and $t_{d}$ is the disturbance time scale), the dispersion equation yields the following simplified expression for the positive real root $\sigma=\operatorname{Re}(\Omega)$ :

$$
\sigma \approx \frac{T(k)}{2\left[1+k^{2}-k^{4} \xi(k)^{2}\right]} \frac{1}{C},
$$

where $T(k)$ is the driving term as defined in the dispersion equation; this approximation is in agreement with the result reported by García and Castellanos ${ }^{49}$ in the uncharged limit. The above equation implies that $\Omega \sim 1 / C \ll 1$, and therefore negligible growth rates will be found provided the viscosity is high. Equation (74) is only correct when $\alpha \gg 1 / C$, i.e., when the electric relaxation time scale $t_{e}$ is much longer than the disturbance time scale $t_{d} \sim t_{c} \Omega$. In the limit $\alpha \lesssim 1 / C$, a different limiting expression is obtained:

$$
a_{o}+a_{1} \Omega C+a_{2}(\Omega C)^{2}=0,
$$

where

$$
\begin{aligned}
a_{o}= & -\frac{1}{2} T(k) \xi(k) G(k) \chi k^{2}\left[k^{2} \xi(k)^{2}-2 \xi(k)-1\right], \\
a_{1}= & T(k)[\beta G(k)-\xi(k)] \\
& +\frac{\chi \xi(k)}{G(k)}\left\{1+\left[2+k^{2} G(k) \xi(k)\right] k^{2} G(k) \xi(k)\right\} \\
& +[2+G(k)] \xi(k) \chi k^{2}\left[k^{2} \xi(k)^{2}-2 \xi(k)-1\right], \\
a_{2}= & 2[\beta G(k)-\xi(k)]\left[k^{4} \xi(k)^{2}-k^{2}-1\right] .
\end{aligned}
$$

\section{Low-viscosity limit: The boundary layer}

Saville $^{2}$ studied the effects of charge relaxation at the interface of a liquid jet and pointed out that, provided the viscosities were low, viscous stresses concentrated within an electrohydrodynamic boundary layer at the interface. Mestel $^{39}$ points out that an interfacial boundary layer is induced by electrical axial forcing, and refers to Melcher and Taylor $^{11}$ for its physical interpretation. Similarly, in their one-dimensional analysis of slender axisymmetric unelectrified liquid jets, in the low-viscosity limit $(C \ll 1)$, García and Castellanos $^{49}$ identified a boundary layer at the interface in the axial velocity profile. Citing previous work by Borkar and Tsamopulos ${ }^{50}$ and Higuera et al., ${ }^{51}$ they hint at the small influence of the boundary layer in terms of global energy dissipation. A boundary layer, however, is bound to exert intense influence on the behavior of interface-driven jets, such as the surfactant-covered viscous threads analyzed by Craster et al. ${ }^{48}$

In the $(C \ll \Omega)$ limit, the dispersion equation converges to the simplified form:

$$
\mathcal{D},=\Omega^{2} \xi(k)+T(k)=0,
$$

an expression fully coincident with the perfect-conductor dispersion equation. This fact was already remarked by Mestel: ${ }^{39}$ "a slightly surprising feature exhibited by poor 


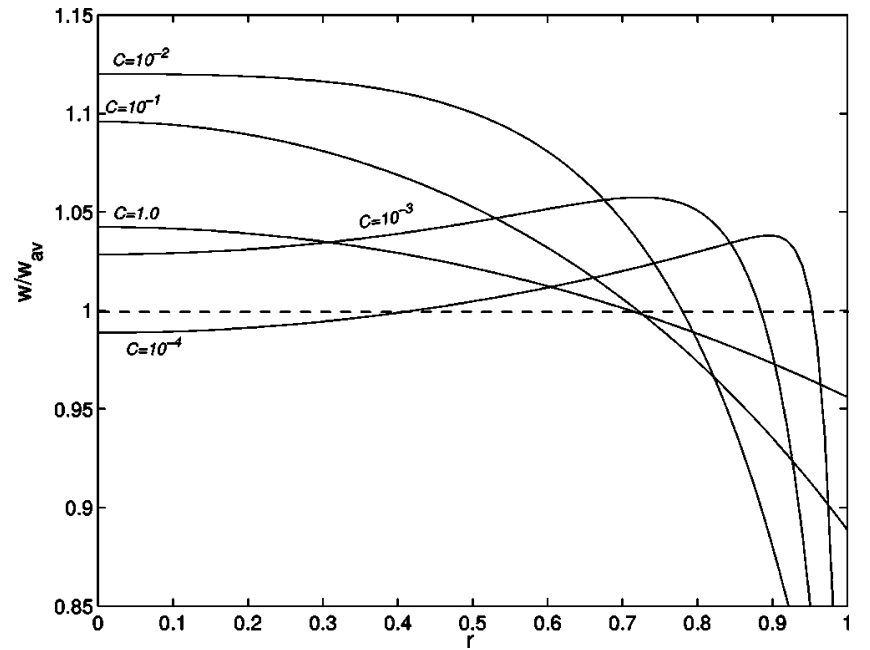

FIG. 10. Radial profiles of the axial velocity, scaled with the average axial velocity, for several values of the Ohnesorge number $\left(C=10^{-4}, C=10^{-3}, C\right.$ $=10^{-2}, C=10^{-1}$, and $C=1.0$ ). The jet is characterized by: $b \gg 1, \beta=10, \chi$ $=0.6$, and $\alpha=1.0$. The wavenumber of the perturbation is $k=0.5$.

conductors is that the small fluid viscosity performs to some extent the role of a high conductivity in permitting the rapid redeployment of surface charge." Therefore, the growth rate depression, however small, caused by imperfect conductivity $(\alpha, \beta \neq \infty)$ vanishes altogether in the proximity of the inviscid limit. Mestel $^{39}$ explained the insensitivity to $\alpha$ and $\beta$ in terms of the rapid redistribution of charge towards the equilibrium state by the boundary layer. In his analysis of a jet flowing in an axial electric field, two cases are considered: the perfectly conducting limit, where the prevailing transport is conduction with a characteristic time $t_{e} \sim \varepsilon_{i} / K$; and the imperfect-conductor range, where the redistribution is due to the surface convection in the boundary layer induced by the electric tangential stress. Our problem however (radial electric field rather than axial field) requires a reassessment of this interpretation.

The effect of the tangential electric stress on the jet dynamics is strongly influenced by the viscosity of the liquid. The shear-induced boundary layer cannot be modeled by a low-order one-dimensional velocity model, inaccuracies arising which grow with decreasing viscosity. The suitability of the average $1 \mathrm{D}$ model in the poor-conductor range, as well as the emergence of the boundary layer, can be illustrated by plotting the radial dependency of the axial velocity for several values of the Ohnesorge number. In Fig. $10(\beta=10, \chi$ $=0.6$ and $\alpha=1.0, k=0.5)$, the axial velocity is scaled with the average velocity $\hat{w}_{\mathrm{av}}=2 i \hat{f} \Omega / k$ and represented as a function of the radius.

Velocity profiles for moderate or high Ohnesorge, $C$ $>10^{-2}$, are convex parabolic. The only exception to the convex profile is to be found when $b$ is very close to 1 , where a concave profile is observed; but this is a situation unrealistic enough in present applications. As the viscosity increases, the profiles become flatter (for $C=10^{-1}$ the maximal difference with respect to the average is about $11 \%$ ). Therefore, an average or parabolic velocity model is adequate for simulating the evolution of jets with moderate or high viscosity.
However, as the viscosity decreases, $C \leqslant 10^{-2}$, the profile develops a boundary-layer structure: (a) a core region, with a very flat concave parabolic profile, and (b) an interface boundary layer with considerable velocity gradient. Velocity departures with respect to the average are above $25 \%(C$ $=10^{-2}$ ), thus confirming the inadequacy of 1D models in this parametric region. Even in the absence of a boundary layer, sharp convex profiles are often observed, thus discouraging routine slice-averaging methods.

The existence of a boundary layer has been postulated by Gañán-Calvo et $a l .{ }^{38}$ to explain the experimental behavior of jets and sprays obtained by electrospraying liquids with small enough viscosity and conductivity, such as light paraffins. In his model (steady jet, tangential electric field), a boundary layer is expected to arise whenever $C(\alpha \beta)^{1 / 3} \ll 1$. Note the implication that the boundary layer disappears in the perfect-conductor limit, i.e., when $\alpha$ or $\beta$ are large enough. A similar observation will be made in our case $\left(\alpha \beta C^{1 / 2} \ll 1\right)$.

The main magnitudes characterizing the boundary layer can be estimated as follows. At the boundary layer, the viscous stresses are of the same order as the tangential electric stress (barred magnitudes are assumed dimensional):

$$
\bar{\tau} \sim \bar{\sigma}_{e} \bar{E}_{t} \sim \mu \frac{\Delta \bar{W}}{\bar{\delta}} \sim \rho \bar{\Omega} \bar{\delta} \bar{W}_{1}
$$

where $\bar{\delta}, \bar{\sigma}_{e}$, and $\bar{\Omega}$ are the physical magnitudes of the width of the boundary layer, the steady surface charge at the interface, and the growth rate or oscillatory frequency, respectively; $\mu$ is the viscosity, $\Delta \bar{W}$ is the velocity jump at the boundary layer, and $\bar{W}_{1}$ is the axial velocity at the interface. $\bar{\sigma}_{e}$ can be estimated as $\varepsilon_{o} E_{o}$.

From the above, the dimensionless width of the boundary layer is $\delta \sim(C / \Omega)^{1 / 2} \sim\left(t_{d} / t_{v}\right)^{1 / 2}$, where $t_{d}$ is the disturbance time scale and $t_{v}$ is the viscous diffusion time. It is interesting to note that, for a given $C$, the width of the boundary layer is sensitive to the main parameters ruling on $\Omega$, i.e., the electrification level $\chi$ and the ground location $b$. Increasing $\chi$, bringing the electrode closer or intensifying the aerodynamic effect, i.e., increasing $\left|U_{\infty}-U_{o}\right|$, gives rise to a narrowing of the boundary layer.

In the long-wavelength limit, $k \ll 1$, the core velocity profile flattens and, in addition, the boundary layer tends to thicken and, thereby, to vanish altogether. However, a persistent boundary layer will be observed provided $C \ll k$ [when $\left.b<b_{o}(\chi)\right]$ or $C \ll k^{2}\left[\right.$ when $\left.b>b_{o}(\chi)\right]$ : see Eqs. (70) and (72).

In addition, the dimensionless stress $\tau=\bar{\tau} /(\gamma / A), A$ being the jet radius and $\gamma$ being the surface tension, is of the order of

$$
\tau \sim \frac{C}{\delta} \frac{\Omega \hat{f}}{k} \sim \frac{C^{1 / 2} \Omega^{3 / 2} \hat{f}}{k},
$$

where the axial velocity jump has been estimated from the average oscillatory axial speed $\hat{w}_{\text {av }}$ whose exact value is $2 i \hat{f} \Omega / k$. This result shows that the tangential stress tends to be negligible when $C \rightarrow 0$. 


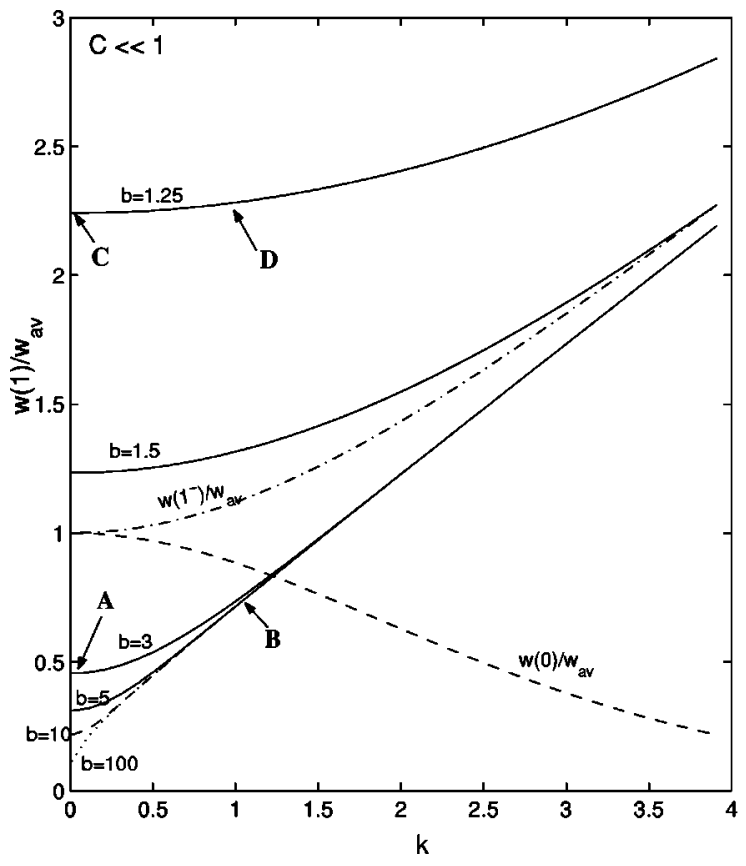

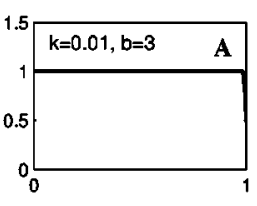
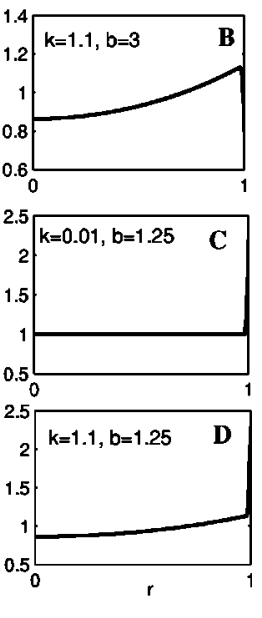

FIG. 11. Main figure: Axial velocity scaled with the average velocity, as a function of the wavelength $k$, for different values of the ground location $b$ in the lowviscosity limit. Also plotted, with the same scaling, are the velocity at the axis $w(0)$ (dashed curve) and the asymptotic core velocity at the interface $w\left(1^{-}\right)$(dash and dot curve). Results are insensitive to all other parameters including $\chi$. Marginal figures: Several representative velocity profiles, as a function of the radius, showing the lazy-shell regime $(b=3)$ and the hyperactive-shell regime $(b=1.25)$ for long and short wavelengths, respectively.
Therefore, the tangential electric field (scaled with $E_{o}$ ) is of the order of

$$
E_{t} \sim \frac{C^{1 / 2} \Omega^{3 / 2} \hat{f}}{k \chi},
$$

and $E_{n}^{i} \sim \lambda E_{t}$. Both $E_{n}^{i}$ and $E_{t}$ are vanishingly small for $C$ $\ll 1$, as happens to be the case in the perfect-conductor limit. The interpretation is straightforward from the above: any departure from the perfect-conductor limit in a radial electric field implies $E_{t} \neq 0$, but a tangential electric stress can only be balanced by a viscous stress. In the $C \ll 1$ limit, the viscous stress is too small to counterbalance any significant tangential field, even in spite of the intensified velocity slope created by the boundary layer. Axial electric fields give rise to quite a different situation. ${ }^{39}$

The radial velocity profile in the low- $C$ limit is therefore insensitive to $\alpha$ and $\beta$. In addition, when the axial velocity is scaled with the average value, self-similarity of the bulk solution is observed not only with respect to $\alpha$ and $\beta$, but also with respect to the electric number $\chi$. The resulting scaled profile is only dependent of the wavelength $k$. The thickness of the boundary layer, however, does depend on all the variables influencing $\Omega$, namely, on $\chi$ and $b$; and the velocity at the interface, $\hat{w}(1) / \hat{w}_{\mathrm{av}}=-1 /[2 G(k, b)]$, depends of $b$. It follows from Eq. (60) that the bulk axial profile (scaled with the average velocity $\hat{w}_{\mathrm{av}}$ ) is characterized by the following values: at the axis, $\hat{w}(0) / \hat{w}_{\mathrm{av}}=k /\left[2 I_{1}(k)\right]$; at the external asymptote $\left(r \rightarrow 1^{-}\right)$of the inviscid core, $\hat{w}\left(1^{-}\right) / \hat{w}_{\mathrm{av}}=k^{2} \xi(k) / 2$. The average velocity is, as in the general case, $2 i \hat{f} \Omega / k$, where $\Omega$, obtained from (79), is dependent of $\chi$ and $b$. The velocity jump at the boundary layer is $\left[\hat{w}(1)-\hat{w}\left(1^{-}\right)\right] / \hat{w}_{\mathrm{av}}=-B k_{v} / 2 \Omega$.

It is worth noting that there is an exception to the above: when $\alpha$ or $\beta$ are sufficiently large, the velocity jump across the boundary layer tends to become zero, while the thickness is preserved. It can indeed be shown that, under the condition that either $\alpha \beta C^{1 / 2} \gg 1, \hat{w}(1) \rightarrow \hat{w}\left(1^{-}\right)$, so that the boundary layer becomes unnoticeable. This confirms that the perfectconductor limit masks the emergence of an interfacial boundary layer, and moderate or small $\alpha$ and $\beta$ values are required for the boundary layer to show up.

The above estimations for the tangential electric field and the interfacial shear, issuing from Eqs. (80), do not hold when $\Delta \bar{W}$, the velocity jump at the boundary layer, and $\bar{W}_{1}$, the axial velocity at the interface, are no longer of the same order. Such is the case in the $\alpha \beta C^{1 / 2} \gg 1$ limit, where $\Delta \bar{W}$ $\rightarrow 0$. However, provided $\alpha \beta \gg 1$, the charge transport balance Eq. (23) shows that the inner electric field will tend to zero, so that $E_{t} \rightarrow 0$. This is the perfect-conductor limit, where the boundary layer is inconspicuous because of the lack of a shear agent.

An interesting feature about the boundary layer is that it either flows more slowly or more rapidly than the inviscid core, as illustrated in Fig. 11, thus defining two regimes which we label as "lazy-shell mode" and "hyperactive-shell mode," respectively.

The discrimination between both modes depends on the electrode position $b$. In the tight-electrode limit $\left(b<b_{o}\right.$ $\approx e^{1 / 2}=1.6487$ ), the boundary layer runs faster than the core; as we separate the electrode, the lazy-shell mode is recovered. The velocity profile tends to be flatter for long wavelengths. In the limit $b \rightarrow \infty$ and $k \rightarrow 0$, an extreme situation is observed, where the interface is stagnant while the core oscillates.

In the above description of a boundary layer, some elements can be highlighted.

(a) Most prior studies concentrate on the boundary layer arising from the action of an axial electric field. It was in this case that a free-jet boundary layer was first reported. ${ }^{11,39}$ Quasi-inviscid jets can only balance tangential stresses by producing a narrow interfacial band with high radial gradients. 
(b) However, from a physical point of view it is not so obvious that a radial electric field will also give rise to a boundary layer. It is here shown that this boundary layer will only become conspicuous when the liquid is a poor conductor. This is a different result, and the following observation is added: the distance at which the electrode is placed gives rise to diverse velocity profiles, with the interface either running faster or slower than the core, while the core velocity profile is insensitive to conductivity and permittivity variables.

(c) This type of boundary layer is based on the competition between the oscillatory acceleration and the viscous term, whereas the axial field boundary layer is based on the balancing of axial stress and viscous term. As such, there is no entrance length nor is there any downstream thickening of the layers; they do not merge. A uniform thickness will be observed in all the lengths of the jet.

\section{1D APPROACH: THE AVERAGE AND THE PARABOLIC APPROXIMATIONS}

One-dimensional models are based on the substitution of a simplified form of the velocity components (a truncated Taylor series in the radial coordinate) into the Navier-Stokes equations. To lowest order in $r$, such methods provide a simplified set of slice equations, widely used in the qualitative analysis of jets. As indicated above, the axial velocity profile tends to become flat as $k$ approaches zero. This is the conceptual basis allowing for an expansion in powers of $r$. However, higher-order approximations (parabolic) are reasonably accurate even for $k \sim 1$ provided the real velocity profile does not exhibit singular patterns such as boundary layers.

Therefore, 1D models fail to converge whenever a boundary layer is present. Such is the case of slightly viscous jets, under the condition that $\alpha \beta C^{1 / 2}$ is, at most, of order 1 . The thickness of the boundary layer, assuming longwavelength disturbances, is approximately $\left[C / B_{1}(b, \chi) k\right]^{1 / 2}$ when $b>b_{o}(\chi)$ and $\left[C / B_{2}(b, \chi) k^{2}\right]^{1 / 2}$ when $b<b_{o}(\chi)$. This implies that, in realistic problems (where $C$ cannot be equal to zero), given any value of $C \ll 1$, a range of $k$ values close to the zero limit can be defined where the boundary layer vanishes and the $1 \mathrm{D}$ approximation holds. In spite of this, 1D models are scarcely useful in the $C \ll 1$ range, because, in the range of wavelengths where maximum growth is observed (ca. $\pi / k_{m}$ ), a distinct boundary-layer pattern is observed.

García and Castellanos ${ }^{49}$ carried out a detailed examination of the equations and their range of validity in the 1D modeling of unelectrified jets. However, their results cannot be extrapolated to charged jets without a general reassessment of the problem.

In this section we derive one-dimensional expressions for the conservation equations. We adopt the slice charge $S$ $=\left(1+F_{z}\right)^{2}{ }^{1 / 2} F \sigma_{e}$ rather than the surface charge density $\sigma_{e} . S$ represents the charge per unit of dimensionless length $z$. Rewritten in terms of $S$, the charge continuity equation (23) becomes

$$
\begin{aligned}
S_{t}+ & \frac{F_{z} U+W}{1+F_{z}^{2}} S_{z}-\alpha \beta F\left(1+F_{z}^{2}\right)^{1 / 2} E_{n}^{i} \\
& -\frac{S}{F\left(1+F_{z}^{2}\right)^{2}}\left(1+3 F_{z}^{2}+2 F_{z}^{4}+F F_{z}^{2} F_{z z}\right) U \\
& +\frac{S F_{z}^{3}}{F\left(1+F_{z}^{2}\right)^{2}}\left(1+F_{z}^{2}+F F_{z z}\right) W \\
& -\frac{S}{1+F_{z}^{2}}\left(U_{r}-F_{z} W_{r}\right)=0 .
\end{aligned}
$$

The one-dimensional approximation is obtained by replacing the 3D velocities $U, W$, and pressure $P$ by

$$
\begin{aligned}
& W(r, z, t)=w_{o}(z, t)+\frac{1}{2} r^{2} w_{2}(z, t)+\cdots, \\
& U(r, z, t)=-\frac{1}{2} r w_{o}^{\prime}(z, t)-\frac{1}{8} r^{3} w_{2}^{\prime}(z, t)+\cdots, \\
& P(r, z, t)=p_{o}(z, t)+\frac{1}{2} r^{2} p_{2}(z, t)+\cdots,
\end{aligned}
$$

where axial derivatives are denoted as primes; time derivatives will be indicated with dots.

The tangential stress balance at the interface (25) is the key equation linking momentum and electric equations. It is from this equation that the convergence of the velocity expansion can be checked. To that end, we observe that axial derivatives are of order $k: \partial / \partial z \sim \lambda^{-1} \sim k$. Therefore,

$$
\begin{aligned}
F w_{2} & -3 w_{o}^{\prime} F^{\prime}-\frac{1}{2} F w_{o}^{\prime \prime}+\frac{1}{6} F^{3} w_{4} \\
& -\left\{\frac{1}{8} F^{3} w_{2}^{\prime \prime}+\frac{7}{4} F^{2} F^{\prime} w_{2}^{\prime}+2 F F^{\prime 2} w_{2}-3 F^{\prime 3} w_{o}^{\prime}\right. \\
& \left.-F F^{\prime 2} w_{o}^{\prime \prime}\right\}+O\left(k^{3}\right) \\
& =\frac{\chi S E_{t}}{F C\left(1+F^{\prime 2}\right)^{1 / 2}} .
\end{aligned}
$$

To leading order, it follows that $w_{2} \approx \chi S E_{t} / C F^{2}$. Note that the electric term in Eq. (87) is left intact while the hydrodynamic part is approximated. This uneven treatment is customary in the literature. By keeping the interfacial electric driving terms unexpanded, an improved accuracy is obtained. The same procedure will be applied to the capillaryelectrical pressure term in the momentum equation below.

In addition, from the Laplace equation in the jet and under the assumption that the main electric forcing is radial, the tangential field (physical magnitude) can be estimated as $\bar{E}_{t} \sim E_{o} / \lambda$, so that $E_{t} \sim k$. It can be inferred that $w_{2} / w_{o} \sim k$, $w_{4} / w_{o} \sim k^{2}$, and so on. However, as will be shown later, $p_{2} / p_{o} \sim k^{2}$.

This is a weaker convergence as in unelectrified problems, where it can be shown ${ }^{49}$ that $w_{2} / w_{o} \sim k^{2}$. In fact, $k^{2}$ convergence is recovered in our case provided the liquid is a perfect dielectric $(S=0)$ or a perfect conductor $(\alpha \beta \gg 1)$. In both cases, the right-hand side of Eq. (87) becomes zero and the tangential stress balance is the same as in the unelectrified limit. In all other cases, $k$ convergence will be observed, and this explains why, in some situations involving poor con- 
ductors, lowest-order 1D models fail to give accurate approximations and at least second-order truncations are required.

Note that the above equation includes three terms in curly brackets which can be neglected to order $\left(k^{3}\right)$. These terms have been retained just for the sake of comparing results with available higher-order expressions in the unelectrified limit. ${ }^{49}$ Indeed, when $\chi=0$, Eq. (87) is accurate with errors $O\left(k^{6}\right)$.

\section{A. Parabolic or $O\left(k^{3}\right)$ approximation}

In this section, the 1D model is taken to order $k^{3}$ by keeping the first two terms in the velocity expansion. This amounts to modeling the axial velocity as a parabolic profile. The continuity equation of the surface charge, the stress balance in the normal direction, and the kinematic condition read

$$
\dot{S}+\left[\left(w_{o}+\frac{1}{2} F^{2} w_{2}\right) S\right]^{\prime}=\alpha \beta F\left(1+F^{\prime 2}\right)^{1 / 2} E_{n}^{i}+O\left(k^{3}\right),
$$

$$
\begin{array}{r}
p_{o}+\frac{1}{2} F^{2} p_{2}=p_{\mathrm{ce}}-C\left(w_{o}^{\prime}+\frac{3}{4} F^{2} w_{2}^{\prime}+2 F F^{\prime} w_{2}\right) \\
+\left\{C\left(F F^{\prime} w_{o}^{\prime \prime}+3 F^{\prime 2} w_{o}^{\prime}\right)\right\}+O\left(k^{3}\right), \\
\dot{F}+\left(w_{o}+\frac{1}{2} F^{2} w_{2}\right) F^{\prime}+\frac{1}{2} F w_{o}^{\prime}+\frac{1}{8} F^{3} w_{2}^{\prime}+O\left(k^{3}\right) ;
\end{array}
$$

the pressure equation includes $O\left(k^{3}\right)$ terms in curly brackets, which are retained with the same purpose as before. These equations are accurate to $O\left(k^{4}\right)$ in the unelectrified limit. The capillary-electrical pressure term is defined as in Eq. (26).

The momentum equations follow from inserting the velocity $r$-expansions and equating terms with the same power in $r$. To order $O\left(k^{2}\right)$ only three equations are required $\left(r^{0}, r^{2}\right.$ terms in the axial direction; leading term in the radial direction):

$$
\begin{aligned}
& \dot{w}_{o}+w_{o} w_{o}^{\prime}=-p_{o}^{\prime}+C\left(w_{o}^{\prime \prime}+2 w_{2}\right), \\
& \dot{w}_{2}+w_{o} w_{2}^{\prime}=-p_{2}^{\prime}+C\left(w_{2}^{\prime \prime}+\frac{4}{3} w_{4}\right), \\
& \dot{w}_{o}^{\prime}+w_{o} w_{o}^{\prime \prime}-\frac{1}{2} w_{o}^{\prime 2}=2 p_{2}+C\left(w_{o}^{\prime \prime \prime}+2 w_{2}^{\prime}\right) .
\end{aligned}
$$

The interfacial stress balance equations are fed into the momentum equations and the pressure components $p_{o}, p_{2}$, as well as $w_{4}$, are eliminated. As a result, two condensedmomentum equations are obtained in replacement of Eqs. (87), (89), and (91)-(93):

$$
\begin{aligned}
\dot{w}_{o}+ & w_{o} w_{o}^{\prime}-\left\{\frac{1}{4} F^{2}\left[\dot{w}_{o}^{\prime}+w_{o} w_{o}^{\prime \prime}-\frac{1}{2} w_{o}^{\prime 2}\right]\right\}^{\prime} \\
= & -p_{\mathrm{ce}}^{\prime}+C\left\{\left(2-4 F^{\prime 2}-F F^{\prime \prime}\right) w_{o}^{\prime \prime}\right. \\
& +2\left(1+F^{\prime 2}+F F^{\prime \prime}\right) w_{2}-\frac{1}{4} F^{2} w_{o}^{i v}-\frac{3}{2} F F^{\prime} w_{o}^{\prime \prime \prime} \\
& \left.+\frac{1}{4} F^{2} w_{2}^{\prime \prime}+\frac{5}{2} F F^{\prime} w_{2}^{\prime}-6 F^{\prime} F^{\prime \prime} w_{o}^{\prime}\right\},
\end{aligned}
$$

$$
\begin{aligned}
\dot{w}_{2}+ & w_{o} w_{2}^{\prime}+\frac{1}{2} \dot{w}_{o}^{\prime \prime}+\frac{1}{2} w_{o} w_{o}^{\prime \prime \prime} \\
= & \frac{8 \chi S E_{t}}{F^{4}\left(1+F^{\prime 2}\right)^{1 / 2}}+\frac{C}{F^{4}}\left\{4 F^{2}\left(1-2 F^{\prime 2}\right) w_{o}^{\prime \prime}\right. \\
& \left.+24 F F^{\prime}\left(1-F^{\prime 2}\right) w_{o}^{\prime}+\frac{1}{2} F^{4} w_{o}^{i v}\right\} \\
& +\frac{C}{F^{4}}\left\{-8 F^{2}\left(1-2 F^{\prime 2}\right) w_{2}+3 F^{4} w_{2}^{\prime \prime}+14 F^{3} F^{\prime} w_{2}^{\prime}\right\} .
\end{aligned}
$$

Up to this point, the equations hold for arbitrary electric field, i.e., axial field, radial field, or other situations are encompassed in the model. Therefore, the parabolic 1D equations above can be used in a wide variety of electrohydrodynamic problems.

Next, we assume small departures of the electric variables with respect to the static solution ( $w_{o}$ and $w_{2}$ are intrinsically small); in addition, the radial electric field assumption is introduced:

$$
\left(\begin{array}{c}
\phi^{o}(z, r, t) \\
\phi^{i}(z, r, t) \\
S(z, t) \\
F(z, t)
\end{array}\right)=\left(\begin{array}{c}
\ln (b / r) \\
\ln (b) \\
1 \\
1
\end{array}\right)+\left(\begin{array}{c}
\widetilde{\phi}^{o}(r, z, t) \\
\widetilde{\phi}^{i}(r, z, t) \\
\tilde{S}(z, t) \\
\tilde{f}(z, t)
\end{array}\right),
$$

and the perturbation is written as

$$
\left(\begin{array}{c}
w_{o}(z, t) \\
w_{2}(z, t) \\
\widetilde{\phi}^{o}(r, z, t) \\
\widetilde{\phi}^{i}(r, z, t) \\
\tilde{S}(z, t) \\
\tilde{f}(z, t)
\end{array}\right)=\operatorname{Re}\left[\left(\begin{array}{c}
-i \hat{w}_{o} \\
-i \hat{w}_{2} \\
\hat{\phi}^{o}(r) \\
\hat{\phi}^{i}(r) \\
\hat{S} \\
\hat{f}
\end{array}\right) \zeta\right],
$$

where $\zeta$ is equal to $e^{\Omega t+i k z}$. In addition, the electric boundary conditions are imposed (here, primes denote radial derivatives):

$$
\begin{aligned}
& -\hat{f}+\hat{\phi}^{o}(1)=\hat{\phi}^{i}(1), \\
& -\left(\hat{\phi}^{o}\right)^{\prime}(1)+\beta\left(\hat{\phi}^{i}\right)^{\prime}(1)=\hat{S}, \\
& \hat{\phi}^{o}(b)=0 .
\end{aligned}
$$

The inner and outer electric fields are exactly modeled as in the 3D description [see Eqs. (61) and (62)]. In addition, the condensed-momentum equations, the charge conservation equation, and the kinematic condition are linearized. The result is a linear system $\mathcal{M}_{2} \cdot \mathbf{x}=0$, where $\mathbf{x}$ $=\left(\hat{w}_{o}, \hat{w}_{2}, \hat{f}, A_{e}, B_{e}, \hat{S}\right)^{T}$ and $\mathcal{M}_{2}$ is a $6 \times 6$ matrix: 


$$
\left(\begin{array}{cccccc}
4 k & k & 8 \Omega & 0 & 0 & 0 \\
C k^{2}\left(8+k^{2}\right)+\Omega\left(4+k^{2}\right) & C\left(-8+k^{2}\right) & 4 k\left(1-k^{2}-\chi\right) & -4 k \chi & 0 & 0 \\
C\left(8 k^{2}-k^{4}\right)-k^{2} \Omega & 2\left[C\left(8+3 k^{2}\right)+\Omega\right] & 16 k \chi & -16 k G \chi & 0 & 0 \\
0 & 0 & 0 & -1 & \beta & -1 \\
0 & 0 & -1 & G & -\xi & 0 \\
k & k / 2 & 0 & 0 & \alpha \beta & \Omega
\end{array}\right)
$$

the rows of $\mathcal{M}_{2}$ containing the kinematic condition, the $w_{o}$ and $w_{2}$ momentum equations, the jump in the normal electric field at the interface, the continuity of potential at the interface and the charge conservation equation. The dispersion equation, $\mathcal{D}_{2}=0$, is readily obtained by setting the determinant of $\mathcal{M}_{2}$ equal to zero.

\section{B. Slice-average approximation}

A more simplified 1D model is widespread in the literature; in this model, the tangential stress balance is truncated with errors $O\left(k^{2}\right)$ :

$F w_{2}-\left\{3 w_{o}^{\prime} F^{\prime}+\frac{1}{2} F w_{o}^{\prime \prime}\right\}+O\left(k^{2}\right)=\frac{\chi S E_{t}}{F C\left(1+F^{\prime 2}\right)^{1 / 2}}$,

implying that $w_{2}=O(k)$. Again, higher-order terms, in curly brackets, have been kept with the purpose of preserving a consistent equation valid with errors $O\left(k^{4}\right)$ in the unelectrified case, where $w_{2}=O\left(k^{2}\right)$. A single condensed-momentum equation is obtained:

$$
\dot{w}_{o}+w_{o} w_{o}^{\prime}=-p_{c e}^{\prime}+3 C \frac{\left(F^{2} w_{o}^{\prime}\right)^{\prime}}{F^{2}}+\frac{2 \chi S E_{t}}{F^{2}\left(1+F^{\prime 2}\right)^{1 / 2}}+O\left(k^{2}\right) .
$$

The kinematic condition is truncated at $O\left(k^{2}\right)$. The remaining equations, boundary conditions, and linearization model are identical as in the parabolic case. The average linear system is now $\mathcal{M}_{1} \cdot \mathbf{y}=0$, where $\mathbf{y}=\left(\hat{w}_{o}, \hat{f}, A_{e}, B_{e}, \hat{S}\right)^{T}$ and $\mathcal{M}_{1}$ is a $5 \times 5$ matrix:

$$
\left(\begin{array}{ccccc}
\hline k & 2 \Omega & 0 & 0 & 0 \\
-\left(\Omega+3 C k^{2}\right) & k\left(-1+k^{2}+\chi\right) & k \chi & 2 k \chi \xi & 0 \\
0 & 0 & -1 & \beta & -1 \\
0 & -1 & G & -\xi & 0 \\
k & 0 & 0 & \alpha \beta+\chi k^{2} \xi / 2 C & \Omega
\end{array}\right)
$$

the rows of $\mathcal{M}_{1}$ containing the kinematic condition, the $w_{o}$ momentum equation, the jump in the normal electric field at the interface, the continuity of potential at the interface and the charge conservation equation. The dispersion equation is $\mathcal{D}_{1}=\operatorname{det}\left(\mathcal{M}_{1}\right)=0$.

This equation can be written in analogy to the 3Ddispersion equation.

$$
\begin{aligned}
\mathcal{D}_{1}= & \frac{2}{k^{2}} \Omega^{2}+6 C \Omega+\Xi_{1}(k)\left(4+4 G(k)+\frac{1}{G(k)}\right) \\
& +T(k)=0,
\end{aligned}
$$

where the auxiliary functions $G(k)$ and $\xi(k)$ and the driving term $T(k)$ are defined as in the 3D-dispersion equation, and

$$
\Xi_{1}(k)=\frac{\chi \xi(k)}{E_{1}(k)}
$$

$$
E_{1}(k)=G(k) \beta\left(1+\frac{\alpha}{\Omega}\right)-\xi(k)+\frac{\chi G(k) \xi(k) k^{2}}{2 \Omega C} .
$$

Apart from the divergent $\Xi$ definition, the average dispersion equation, $\mathcal{D}_{1}=0$, can be easily deduced from the general one, Eq. (63), by assuming $k \ll 1, k_{v} \ll 1$, and observing that $k^{2} \xi(k) \rightarrow 2$ when $k \rightarrow 0$. The same simplifications as in the $3 \mathrm{D}$ case can be obtained in different parametric extremes. For instance, assuming low viscosity $(C \ll 1)$,

$$
\mathcal{D}_{1}=\frac{2}{k^{2}} \Omega^{2}+T(k)=0 ;
$$

an identical expression is obtained when the jet is a perfect conductor $\left(\alpha \beta C^{1 / 2} \gg 1\right)$. In the opposite limit $(C \gg 1)$, the positive real root of the dispersion equation is

$$
\sigma=-\frac{T(k)}{6 C} .
$$



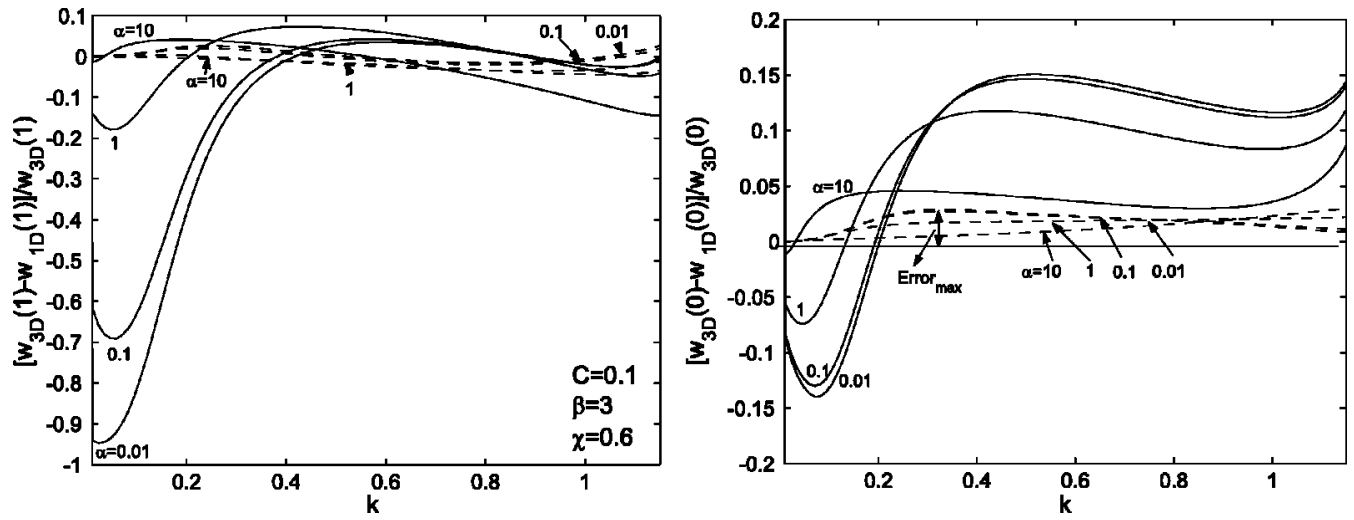

FIG. 12. Scaled difference between the 3D and 1D axial velocity $\left[w(r)-w_{1 \mathrm{D}}(r)\right] / w(r)$ at the interface $(r=1)$ and at the axis $(r=0)$ in the average and parabolic (dashed line) approximation, for different values of $k$ and $\alpha(\beta=3, C=0.1, \chi=0.6, b \gg 1)$.

\section{Assessment of the validity of 1D models}

The simplifications inherent to $1 \mathrm{D}$ models tend to become unacceptable whenever the axial velocity profile departs significantly from the flat or parabolic curve. Such is the case of low- $C$ jets, particularly when $\alpha$ and $\beta$ are small or moderate. Figure 12 shows the scaled difference between the 3D and 1D axial velocity $\left[w_{3 \mathrm{D}}(r)-w_{1 \mathrm{D}}(r)\right] / w_{3 \mathrm{D}}(r)$ at the interface $(r=1)$ and at the axis $(r=0)$ in the average and parabolic approximation, for different values of $k$ and $\alpha(\beta$ $=3, C=0.1, \chi=0.6, b \gg 1)$. Provided $\alpha$ is small enough, significant errors can be noticed in the average model. These errors are smoothed out with the parabolic approximation.

The maximum relative error $\operatorname{Max}\left\{\left[w_{3 \mathrm{D}}(r)\right.\right.$ $\left.\left.-w_{1 \mathrm{D}}(r)\right] / w_{3 \mathrm{D}}(r)\right\}$ of the 1D approximation at $r=0$ and $r=1$, for arbitrary $k$ and different values of $C$ and $\alpha C^{1 / 2}(\beta=3, \chi$ $=0.6, b \gg 1)$ is shown in Fig. 13. Considerable errors can be observed in the low- $C$ limit, but the parabolic approximation achieves a better fit.

Figure 14 shows different radial velocity profiles for variable $\alpha C^{1 / 2}$. The 3D solution exhibits a sharp boundarylayer pattern, which becomes abortive as $\alpha C^{1 / 2} \rightarrow \infty$. The parabolic approximation tries to adhere to the interfacial velocity, and fails to describe intermediate values of $r$. Nevertheless, since the key physical phenomena take place at the interface, the parabolic model will be substantially more accurate than the average model, given its fidelity to the interfacial speed.

Finally, Fig. 15 shows growth rate curves as obtained with the 3D equations and compares them to the average and parabolic 1D approximations. In the absence of a boundary layer $(C=1)$, the parabolic approximation ensures improved accuracy, particularly in the long-wavelength range. However, very low Ohnesorge numbers give rise to a double peak axial velocity profile, and both orders of approximation fail to describe accurately the growth behavior.

\section{CONCLUSIONS}

The present paper can be considered as an investigation on the shortcomings of the perfect-conductor hypothesis in electrified jets. This is a widely used assumption in most of the electrospray literature; with perfect conductors, the electric stress is perpendicular to the interface, a hypothesis that

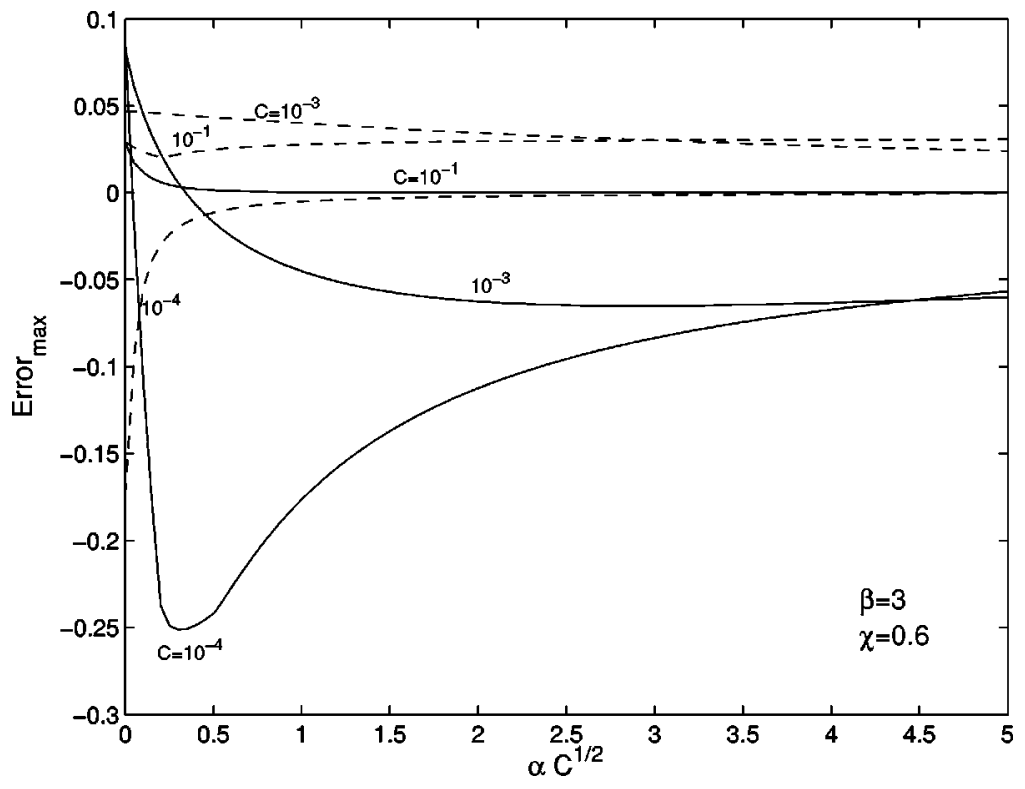

FIG. 13. Maximum relative error of the parabolic 1D approximation at the axis $(r=0)$ and at the interface $(r$ $=1$, dashed line) $\operatorname{Max}\left\{\left[w_{3 \mathrm{D}}(r)-w_{1 \mathrm{D}}(r)\right] / w_{3 \mathrm{D}}(r)\right\}$, arbitrary $k$, plotted for different values of $\alpha$ and $\alpha C^{1 / 2}(\beta$ $=3, \chi=0.6, b \gg 1)$. 


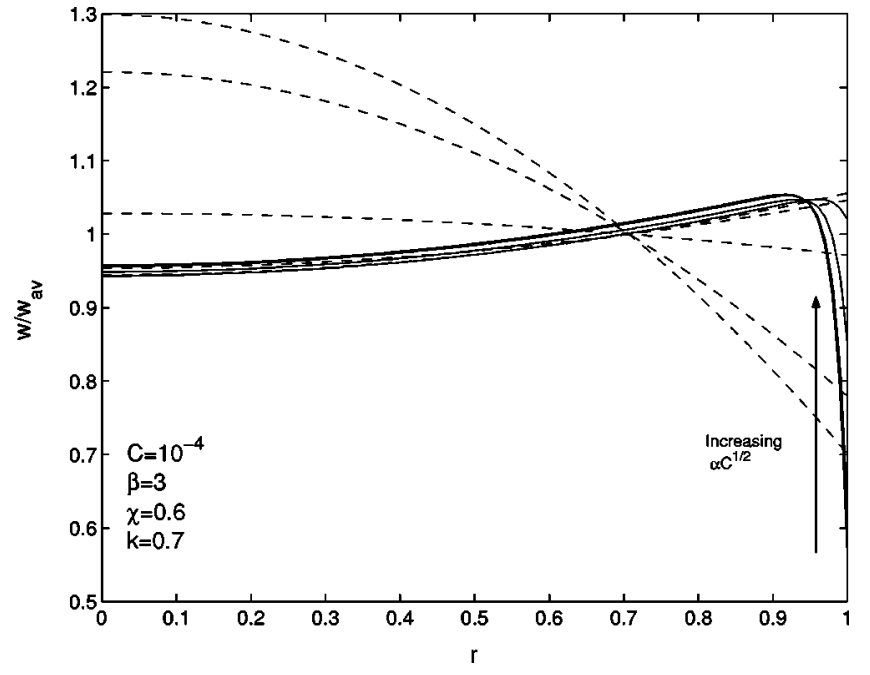

FIG. 14. Radial profiles of the axial velocity, scaled with the average axial velocity, for several values of $\alpha C^{1 / 2}$. The 3D solution, exhibiting a clear boundary-layer pattern for moderate or small $\alpha C^{1 / 2}$, ranging from 0.001 to 10 , is shown together with the parabolic 1D approximation (dashed line): $b \gg 1, \beta=3, k=0.7, \chi=0.6$, and $C=0.0001$.

cannot be preserved when dealing with leaky dielectrics. ${ }^{9}$ In addition, lowest-order 1D models, assuming a flat axial velocity profile, are assessed and found defective whenever $C$ is sufficiently small (an interfacial boundary layer emerges) or in cases where the real velocity profile is sharply convex. This latter situation is enhanced by finite permittivity and conductivity liquids. Moderate and low $\alpha$ and $\beta$ are shown to be abundant in the literature and applications of electrified jets. In such cases, the routine adoption of an average model may be misleading, and parabolic or 3D models become mandatory.

The following main conclusions are drawn.

(a) A general dispersion equation has been obtained for axisymmetric perturbations modeling the influence of conductivity, permittivity, electric field, viscosity, surface tension, electrode distance, and ambient velocity. Different parametric limits are explored: longwavelength, high and low viscosity, charge-convection dominant or negligible. The growth factor is insensitive to $\alpha$ and $\beta$ in the low- $C$ limit. In the general case, as $\alpha$ and $\beta$ grow, the perfect-conductor limit is recovered.
(1) When the viscosity is sufficiently small, $C \ll \Omega$, i.e., the viscous diffusion time scale $t_{v}$ is much longer than the disturbance time scale $t_{d}$, an interfacial boundary layer, driven by electric shear, is observed. At the inside, the boundary layer borders with the inviscid jet core, where the axial velocity profile follows a self-similar trend, insensitive to all electric parameters $\alpha, \beta$, and $\chi$. In spite of the increased velocity gradient at the boundary layer, viscous stresses become vanishingly small in the $C \rightarrow 0$ limit. The occurrence of a boundary layer is a key event in interface-driven jets, and it is only through finite conductivity and permittivity that the boundary layer comes to be fully deployed.

(2) Depending on the location of the ground electrode, the boundary layer either runs faster than the core $\left(b>b_{1}\right.$ $\left.\approx e^{1 / 2}\right)$ or lags behind it $\left(b<b_{1}\right)$. Substantial velocity differences can be observed, particularly for shortwavelength disturbances: therefore caution is required when modeling surface charge transport.

(3) Liquids with high conductivity or high dielectric constant $\left(\alpha \beta C^{1 / 2} \gg 1\right)$ give rise to an electric field perpendicular to the interface (zero-tangential field) and therefore weaken the boundary layer. In the perfect-conductor limit, the boundary-layer velocity jump drops to zero. This may explain why the boundary layer has been disregarded in most of the state-of-the-art studies on the subject.

(4) Inviscid jets cannot balance electric shear stresses at the interface and therefore behave as perfect conductors. The emergence of a boundary layer gives rise to an axial velocity profile that cannot be modeled accurately with low-order expansions in terms of the radius.

(5) Increasing convexity in the axial velocity profile is observed when $\alpha$ or $\beta$ decrease, inviting adoption of higher-order expansions rather than average models.

(b) Slice equations, based on the approximation of the axial velocity profile as an expansion in powers of the radius, are obtained to lowest order (average 1D) and second order (parabolic 1D). The expressions obtained hold for arbitrary electric field.

(1) Significant errors introduced by the lowest-order 1D approximation (widely used in the literature) may be masked by the prevalence of large conductivity and per-
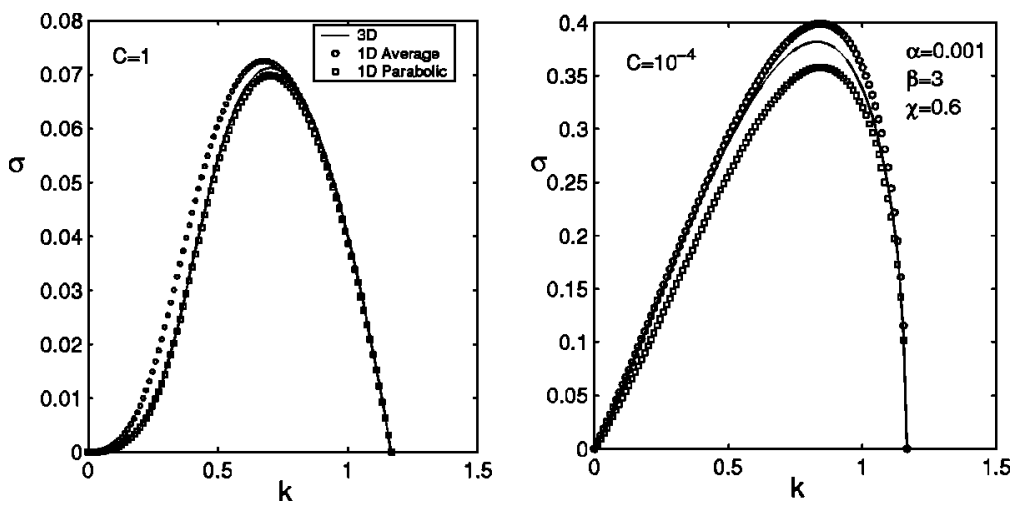

FIG. 15. Growth rate $\sigma$ vs the wavenumber $k$ for a jet with $b \gg 1, \alpha=0.001, \beta=3, \chi=0.6$, and several Ohnesorge numbers $C=1, C=0.0001$. The average and parabolic approximations are plotted as dot curves. 
mittivity liquids in most reported studies. A realistic assessment of $\alpha$ and $\beta$ leads to questioning the validity of average approximations, especially in their description of interfacial magnitudes. In effect, the accuracy of the interfacial velocity as described by the 1D average model is poor in a wide parametric range provided $\alpha$ and $\beta$ are moderate.

\section{ACKNOWLEDGMENTS}

This work was partially supported by the Spanish Ministry of Science and Technology under Grant No. DPI200204305-C02-02. One of the authors (P.R.-C.) wishes to thank the regional Government of Andalusia, for the support received through a Return Grant No. 72/2003.

\section{APPENDIX: DERIVATION OF THE DISPERSION RELATION}

The dispersion relation can be routinely obtained by equating to zero the $7 \times 7$ determinant of the algebraic equations corresponding to the boundary conditions at the interface (50)-(57). However, arriving at the simplified expression reported in this paper is not straightforward. A brief note on the procedure followed (in the simplified case where the gas influence can be neglected) is therefore appended for reference:

$$
\begin{aligned}
& -\hat{f}+\mathcal{A}_{e} G(k)=\mathcal{B}_{e} \xi(k), \\
& \hat{\sigma}_{e}=-\left(\hat{f}+\mathcal{A}_{e}\right)+\beta \mathcal{B}_{e}, \\
& \Omega \hat{\sigma}_{e}=-\alpha \beta \mathcal{B}_{e}+\mathcal{A}\left[k^{2} \xi(k)-1\right]-\mathcal{B}\left[k_{v}^{2} \xi\left(k_{v}\right)-1\right], \\
& \mathcal{A}-\mathcal{B}-\Omega \hat{f}=0, \\
& \mathcal{A}\left\{\Omega \xi(k)+2 C\left[k^{2} \xi(k)-1\right]\right\}-\mathcal{B}\left\{2 C\left[k_{v}^{2} \xi\left(k_{v}\right)-1\right]\right\} \\
& \quad+\left(-1+k^{2}\right) \hat{f}+\chi\left(\hat{f}+\mathcal{A}_{e}\right)=0,
\end{aligned}
$$

and

$$
\mathcal{A}-\mathcal{B}\left(1+\frac{\Omega}{2 k^{2} C}\right)+\frac{\chi}{2 C} \xi(k) \mathcal{B}_{e}=0
$$

where the auxiliary functions $G$ and $\xi$ are defined as in (65).

Using Eqs. (A1)-(A3), $\mathcal{A}_{e}$ and $\mathcal{B}_{e}$ can be expressed as a function of $\mathcal{A}$ and $\mathcal{B}$

$$
\begin{aligned}
\mathcal{A}_{e}= & \frac{\hat{f}}{E}\left[\beta\left(1+\frac{\alpha}{\Omega}\right)+\xi(k)\right]+\frac{\xi(k)}{E \Omega}\left\{\mathcal{A}\left[k^{2} \xi(k)-1\right]\right. \\
& \left.-\mathcal{B}\left[k_{v}^{2} \xi\left(k_{v}\right)-1\right]\right\}
\end{aligned}
$$

and

$$
\begin{aligned}
\mathcal{B}_{e}= & \frac{\hat{f}}{E}[1+G(k)]+\frac{G(k)}{E \Omega} \\
& \times\left\{\mathcal{A}\left[k^{2} \xi(k)-1\right]-\mathcal{B}\left[k_{v}^{2} \xi\left(k_{v}\right)-1\right]\right\},
\end{aligned}
$$

where $E$ is defined as in (66).
Substituting these expressions in (A5) and (A6) we get

$$
\begin{aligned}
& \mathcal{A}\left[\Omega \xi(k)+\left(2 C+\frac{\Xi(k)}{\Omega}\right)\left[k^{2} \xi(k)-1\right]\right] \\
& \quad-\mathcal{B}\left[\left(2 C+\frac{\Xi(k)}{\Omega}\right)\left[k_{v}^{2} \xi\left(k_{v}\right)-1\right]\right] \\
& \quad+\hat{f}\left[k^{2}-1+[\chi+\Xi(k)]\left(1+\frac{1}{G(k)}\right)\right]=0
\end{aligned}
$$

and

$$
\begin{aligned}
\mathcal{A}[1 & \left.+\frac{\Xi(k)}{2 C \Omega} G(k)\left[k^{2} \xi(k)-1\right]\right] \\
& -\mathcal{B}\left[1+\frac{\Omega}{2 k^{2} C}+\frac{\Xi(k)}{2 C \Omega} G(k)\left[k_{v}^{2} \xi\left(k_{v}\right)-1\right]\right] \\
& +\hat{f} \frac{\Xi(k)}{2 C}[G(k)+1]=0,
\end{aligned}
$$

where $\Xi$ is defined as in (66).

$\mathcal{A}$ and $\mathcal{B}$ can be expressed in terms of $\hat{f}$ using (A10) and (A4)

$$
\mathcal{A}=\hat{f} \frac{\Omega+2 k^{2} C+\frac{\Xi(k)}{\Omega} k^{2}\left[1+G(k) k_{v}^{2} \xi\left(k_{v}\right)\right]}{1+\frac{\Xi(k)}{\Omega^{2}} k^{2} G(k)\left[k_{v}^{2} \xi\left(k_{v}\right)-k^{2} \xi(k)\right]}
$$

and

$$
\mathcal{B}=\hat{f} \frac{2 k^{2} C+\frac{\Xi(k)}{\Omega} k^{2}\left[1+G(k) k^{2} \xi(k)\right]}{1+\frac{\Xi(k)}{\Omega^{2}} k^{2} G(k)\left[k_{v}^{2} \xi\left(k_{v}\right)-k^{2} \xi(k)\right]},
$$

and substituting both (A11) and (A12) in (A9), the dispersion relation (63) is obtained.

${ }^{1}$ J. R. Melcher, Field-Coupled Surface Waves (MIT Press, Cambridge, MA, 1963).

${ }^{2}$ D. A. Saville, "Stability of electrically charged viscous cylinders," Phys. Fluids 14, 1095 (1971).

${ }^{3}$ A. L. Huebner and H. N. Chu, "Instability and break up of charged liquid jets," J. Fluid Mech. 49, 361 (1971).

${ }^{4}$ E. R. Setiawan and S. D. Heister, "Nonlinear modeling of an infinite electrified jet," J. Electrost. 42, 243 (1997).

${ }^{5} \mathrm{~K}$. Baudry, H. Romat, and G. Artana, "Theoretical influence of the pressure of the surrounding atmosphere on the stability of high velocity jets," J. Electrost. 40, 73 (1997).

${ }^{6}$ F. J. García, "Aplicación de modelos unidimensionales a la dinámica de columnas líquidas con y sin campo eléctrico," Ph.D. thesis, Universidad de Sevilla, 1998.

${ }^{7}$ H. González, F. J. García, and A. Castellanos, "Stability analysis of conducting jets under ac radial electric fields for arbitrary viscosity," Phys. Fluids 15, 395 (2003).

${ }^{8}$ A. B. Basset, "Waves and jets in a viscous liquid," Am. J. Math. 16, 93 (1894).

${ }^{9}$ D. A. Saville, "Electrohydrodynamics: The Taylor-Melcher leaky dielectric model," Annu. Rev. Fluid Mech. 29, 27 (1997).

${ }^{10}$ G. I. Taylor, "Electrically driven jets," Proc. R. Soc. London, Ser. A 313, 453 (1969).

${ }^{11}$ J. R. Melcher and G. I. Taylor, "Electrohydrodynamics: A review of the role of interfacial shear stresses," Annu. Rev. Fluid Mech. 1, 111 (1969).

${ }^{12}$ D. A. Saville, "Electrohydrodynamic stability: Fluid cylinders in longitudinal electric fields," Phys. Fluids 13, 2987 (1970). 
${ }^{13}$ D. A. Saville, "Electrohydrodynamic stability: Effects of charge relaxation at interface of a liquid jet," J. Fluid Mech. 48, 815 (1971).

${ }^{14}$ C. L. Burcham and D. A. Saville, "The electrohydrodynamic stability of a liquid bridge: microgravity experiments on a bridge suspended in a dielectric gas," J. Fluid Mech. 405, 37 (2000).

${ }^{15}$ C. L. Burcham and D. A. Saville, "Electrohydrodynamic stability: TaylorMelcher theory for a liquid bridge suspended in a dielectric gas," J. Fluid Mech. 452, 163 (2002).

${ }^{16}$ A. M. Gañan-Calvo, "Generation of steady liquid microthreads and micron-sized monodisperse sprays in gas streams," Phys. Rev. Lett. 80, 285 (1998).

${ }^{17}$ J. M. López-Herrera, A. M. Gañán-Calvo, and M. Perez-Saborid, "Onedimensional simulation of the breakup of capillary jets of conducting liquids. Application to e.h.d. spraying," J. Aerosol Sci. 30, 895 (1999).

${ }^{18}$ O. E. Yildirim and O. A. Basaran, "Deformation and breakup of stretching bridges of Newtonian and shear-thinning liquids: Comparison of one- and two-dimensional models," Chem. Eng. Sci. 56, 211 (2001).

${ }^{19}$ J. M. López-Herrera and A. M. Gañán-Calvo, "A note on charged capillary jet breakup of conducting liquids. Experimental validation of a viscous one dimensional model," J. Fluid Mech. 501, 303 (2004).

${ }^{20}$ J. B. Keller, S. I. Rubinow, and Y. O. Tu, "Spatial instability of a jet," Phys. Fluids 16, 2052 (1973).

${ }^{21}$ G. Artana, G. Touchard, and H. Romat, "Absolute and convective instabilities in an electrified jet," J. Electrost. 40-41, 33 (1997).

${ }^{22}$ B. O'Donnell, J. N. Chen, and S. P. Lin, "Transition from convective to absolute instability in a liquid jet," Phys. Fluids 13, 2732 (2001).

${ }^{23}$ A. Chauhan, C. Maldarelli, D. S. Rumschitzki, and D. T. Papageorgiou, "An experimental investigation of the convective instability of a jet," Chem. Eng. Sci. 58, 2421 (2003).

${ }^{24}$ A. Kalaaji, B. Lopez, P. Attane, and A. Soucemarianadin, "Breakup length of forced liquid jets," Phys. Fluids 15, 2469 (2003).

${ }^{25}$ S. P. Lin and R. D. Reitz, "Drop and spray formation from a liquid jet," Annu. Rev. Fluid Mech. 30, 85 (1998).

${ }^{26} \mathrm{~S}$. P. Lin and J. N. Chen, "Role played by the interfacial shear in the instability mechanism of a viscous liquid jet surrounded by a viscous gas in a pipe," J. Fluid Mech. 376, 37 (1998).

${ }^{27}$ J. M. Gordillo, M. Pérez-Saborid, and A. M. Gañán-Calvo, "Linear stability of co-flowing liquid-gas jets," J. Fluid Mech. 448, 23 (2001).

${ }^{28}$ A. M. Gañán-Calvo, "Cone-jet analytical extension of Taylor's electrostatic solution and the asymptotic universal scaling laws in electrospraying," Phys. Rev. Lett. 79, 217 (1997).

${ }^{29}$ R. P. A. Hartman, D. J. Brunner, D. M. A. Camelot, J. C. M. Marijnissen, and B. Scarlett, "Electrohydrodynamic atomization in the cone-jet mode physical modeling of the liquid cone and jet," J. Aerosol Sci. 30, 823 (1999).

${ }^{30}$ M. Gamero-Castaño and V. Hruby, "Electric measurements of charged sprays emitted by cone-jets," J. Fluid Mech. 459, 245 (2002).
${ }^{31}$ F. J. Higuera, "Flow rate and electric current emitted by a Taylor cone," J. Fluid Mech. 484, 303 (2003).

${ }^{32}$ G. Artana, H. Romat, and G. Touchard, "Theoretical analysis of linear stability of electrified jets flowing at high velocity inside a coaxial electrode," J. Electrost. 43, 83 (1998).

${ }^{33} \mathrm{M}$. Cloupeau and B. Prunet-Foch, "Electrostatic spraying of liquids in cone-jet mode," J. Electrost. 22, 135 (1989).

${ }^{34}$ J. M. Grace and J. C. M. Marijnissen, "A review of liquid atomization by electrical means," J. Aerosol Sci. 25, 1005 (1994).

${ }^{35}$ A. M. Gañán-Calvo, "On the theory of electrohydrodynamically driven capillary jets, J. Fluid Mech. 335, 165 (1997).

${ }^{36}$ A. M. Gañán-Calvo, "The surface charge in electrospraying: Its nature and its universal scaling law," J. Aerosol Sci. 30, 863 (1999).

${ }^{37}$ J. Fernández de la Mora and I. G. Loscertales, "The current emitted by highly conducting Taylor cones,” J. Fluid Mech. 260, 155 (1994).

${ }^{38}$ A. M. Gañán-Calvo, J. Dávila, and A. Barrero, "Current and droplets size in the electrospraying of liquid. Scaling laws," J. Aerosol Sci. 28, 249 (1997).

${ }^{39}$ A. J. Mestel, "Electrohydrodynamic stability of a slightly viscous jet," J. Fluid Mech. 274, 93 (1994).

${ }^{40}$ A. J. Mestel, "Electrohydrodynamic stability of a highly viscous jet," J. Fluid Mech. 312, 311 (1996)

${ }^{41}$ R. P. A. Hartman, D. J. Brunner, D. M. A. Camelot, J. C. M. Marijnissen, and B. Scarlet, "Jet break-up in elelectrohydrodynamic atomization in the cone-jet mode," J. Aerosol Sci. 31, 65 (2000).

${ }^{42}$ S. P. Lin and R. Webb, "Nonaxisymmetric evanescent waves in a viscous liquid jet," Phys. Fluids 6, 2545 (1994).

${ }^{43}$ A. M. Sterling and C. A. Sleicher, "The instability of capillary jets," J. Fluid Mech. 68, 477 (1975).

${ }^{44}$ M. M. Hohman, M. Shin, G. Rutledge, and M. P. Brenner, "Electrospinning and electrically forced jets. I. Stability theory," Phys. Fluids 13, 2201 (2001).

${ }^{45}$ S. Chandrasekhar, Hydrodynamic and Hydromagnetic Stability, 1st. ed. (Clarendon, Oxford, 1961).

${ }^{46}$ Lord Rayleigh, "On the instability of a cylinder of viscous liquid under capillary forces," Philos. Mag. 34, 145 (1892).

${ }^{47}$ J. Eggers, "Nonlinear dynamics and breakup of free-surface flows," Rev. Mod. Phys. 69, 865 (1997).

${ }^{48}$ R. V. Craster, O. K. Matar, and D. T. Papageorgiou, "Pinchoff and satellite formation in surfactant covered viscous threads," Phys. Fluids 14, 1365 (2002).

${ }^{49}$ F. J. García and A. Castellanos, "One-dimensional models for slender axisymmetrical viscous-liquid jets," Phys. Fluids 6, 2676 (1994).

${ }^{50} \mathrm{~A}$. Borkar and J. Tsamopulos, "Boundary-layer analysis of the dynamics of axisymmetric bridges," Phys. Fluids A 3, 2866 (1991).

${ }^{51}$ M. Higuera, J. A. Nicolás, and J. M. Vega, "Linear oscillations of weakly dissipative axisymmetric liquid bridges," Phys. Fluids 6, 438 (1994). 